\title{
The transition density of Brownian motion killed on a bounded set
}

\author{
Kôhei UCHIYAMA \\ Department of Mathematics, Tokyo Institute of Technology \\ Oh-okayama, Meguro Tokyo 152-8551 \\ e-mail: uchiyama@math.titech.ac.jp
}

AMS Subject classification (2010): Primary 60J65; Secondary 35K20

key words: heat kernel; exterior domain; transition probability

\begin{abstract}
We study the transition density of a standard two-dimensional Brownian motion killed when hitting a bounded Borel set $A$. We derive the asymptotic form of the density, say $p_{t}^{A}(\mathbf{x}, \mathbf{y})$, for large times $t$ and for $\mathbf{x}$ and $\mathbf{y}$ in the exterior of $A$ valid uniformly under the constraint $|\mathbf{x}| \vee|\mathbf{y}|=O(t)$. Within the parabolic regime $|\mathbf{x}| \vee|\mathbf{y}|=$ $O(\sqrt{t})$ in particular $p_{t}^{A}(\mathbf{x}, \mathbf{y})$ is shown to behave like $4 e_{A}(\mathbf{x}) e_{A}(\mathbf{y})(\lg t)^{-2} p_{t}(\mathbf{y}-\mathbf{x})$ for large $t$, where $p_{t}(\mathbf{y}-\mathbf{x})$ is the transition kernel of the Brownian motion (without killing) and $e_{A}$ is the Green function for the 'exterior of $A$ ' with a pole at infinity normalized so that $e_{A}(\mathbf{x}) \sim \lg |\mathbf{x}|$. We also provide fairly accurate upper and lower bounds of $p_{t}^{A}(\mathbf{x}, \mathbf{y})$ for the case $|\mathbf{x}| \vee|\mathbf{y}|>t$ as well as corresponding results for the higher dimensions.
\end{abstract}

\section{Introduction and main results}

Let $A \subset \mathbf{R}^{d}, d \geq 2$ be a bounded and non-polar Borel set and $A^{r}$ the set of all regular points of $A$. Denote by $\Omega_{A}$ the unbounded (fine) component of $\mathbf{R}^{d} \backslash A^{r}$. Let $p_{t}^{A}(\mathbf{x}, \mathbf{y})$, $t>0, \mathbf{x}, \mathbf{y} \in \Omega_{A}$ be the transition density of a $d$-dimensional standard Brownian motion in $\Omega_{A}$ killed when it hits $A$. In this paper we obtain an exact asymptotic form of $p_{t}^{A}(\mathbf{x}, \mathbf{y})$ as $t \rightarrow \infty$ that holds true uniformly for $\mathbf{x}, \mathbf{y}$ subject to the constraint $|\mathbf{x}|,|\mathbf{y}|=O(t)$. It is shown by Collet, Martinez and Martin [4] (in which $A$ is assumed to be compact) that as $t \rightarrow \infty$

$$
p_{t}^{A}(\mathbf{x}, \mathbf{y}) \sim \frac{2 u(\mathbf{x}) u(\mathbf{y})}{\pi(\lg t)^{2} t} \quad \text { if } \quad d=2
$$

and

$$
p_{t}^{A}(\mathbf{x}, \mathbf{y}) \sim \frac{u(\mathbf{x}) u(\mathbf{y})}{(2 \pi t)^{d / 2}} \quad \text { if } \quad d \geq 3
$$

uniformly for $\mathbf{x}, \mathbf{y}$ in any compact set of $\Omega_{A}$. Here $u(\mathbf{x})$ is the unique harmonic function in $\Omega_{A}$ that tends to zero as $\mathbf{x}$ approaches $A^{r}$ and is asymptotically equivalent to $\lg |\mathbf{x}|$ or 1 as $|\mathbf{x}| \rightarrow \infty$ according as $d=2$ or $d \geq 3$; and the symbol $\sim$ designates the asymptotic equivalence: the ratio approaches unity in any specified limit. On the other hand it is readily verified that if $\mathbf{x}$ and $\mathbf{y}$ are both sufficiently far from the origin (depending on $t$ when $d=2$ ) and not located in the opposite direction relative to $A$ (in a loose sense), then

$$
p_{t}^{A}(\mathbf{x}, \mathbf{y}) \sim p_{t}^{(d)}(|\mathbf{y}-\mathbf{x}|)
$$


where $|\mathbf{x}|$ denotes the Euclidian length of $\mathbf{x} \in \mathbf{R}^{d}$ and

$$
p_{t}^{(d)}(x)=\frac{1}{(2 \pi t)^{d / 2}} e^{-x^{2} / 2 t} \quad(x \geq 0) .
$$

In the case $d \geq 3$ formula (2) would be naturally extended to (3) for unbounded $\mathbf{x}$ and $\mathbf{y}$ since $u(\mathbf{x}) \rightarrow 1$ as $|\mathbf{x}| \rightarrow \infty$ (although there still remains a problem, the validity of (3) being not obvious when $\mathbf{x}$ and $\mathbf{y}$ in the opposite direction), whereas in the case $d=2$ there is a serious gap between (11) and (3) and it is not clear how they are linked together. Our result, stated below, extends formula (11) by allowing $\mathbf{x}$ or $\mathbf{y}$ to become indefinitely large along with $t$ and makes precise a regime of $t, \mathbf{x}, \mathbf{y}$ for which (3) is valid, and thereby fill the gap between (1) and (3). The asymptotic form in the parabolic regime $|\mathbf{x}|=O(\sqrt{t})$ and/or $|\mathbf{y}|=O(\sqrt{t})$ would particularly be both interesting and important for an obvious reason: our killed Brownian motion starting at any fixed point outside $A$ must be located, at a large time $t$, inside the annulus $M^{-1} \sqrt{t}<|\mathbf{x}|<M \sqrt{t}$ with a high probability if the constant $M$ is large.

The present investigation is motivated by the study of heat equation in $\Omega_{A}$ in which a fundamental role is played by the caloric measure (the harmonic measure of the heat operator $\frac{1}{2} \Delta-(\partial / \partial t)$ in the space-time cylinder $\left.\Omega_{A} \times[0, \infty)\right)$, which consists of two parts: one is the transition probability $p_{t}^{A}(\mathbf{x}, \mathbf{y})|d \mathbf{y}|$, the part on the initial boundary $\Omega_{A} \times\{0\}$ and the other, the lateral part, is the hitting distribution in space-time of the boundary $\partial A$. Here $|\cdot|$ designates the Lebesgue measure on $\mathbf{R}^{d}$ and $\partial A$ the Euclidean boundary of $A$. The asymptotic form of the lateral part is computed in [12] and our present result together with it identifies the explicit asymptotic form of the caloric measure for large time valid uniformly at least in the regime $|\mathbf{x}| \vee|\mathbf{y}|=o(t)$, where $x \vee y=\max \{x, y\}$.

Let $U(a) \subset \mathbf{R}^{d}$ denote the $d$-dimensional open ball centered at the origin with radius $a>0$. $P_{\mathbf{x}}$ denotes the law of a Brownian motion $\left(B_{t}\right)$ started at $\mathbf{x}$. For a bounded Borel set $A, \sigma_{A}$ denotes the first hitting time of $A$ by $B_{t}$, namely $\sigma_{A}=\inf \left\{t>0: B_{t} \in A\right\}$, and $A^{r}$ the set of all regular points of $A$, namely the set of $\mathbf{y}$ such that $P_{\mathbf{y}}\left[\sigma_{A}=0\right]=1$. Put $R_{A}=\sup \left\{|\mathbf{z}|: \mathbf{z} \in A^{r}\right\}$. The set $\Omega_{A}$ is then defined by

$$
\Omega_{A}=\left\{\mathbf{x} \in \mathbf{R}^{d}: P_{\mathbf{x}}\left[\sigma_{\partial U(R)}<\sigma_{A}\right]>0\right\}
$$

with any/some $R>R_{A}$, and the transition density $p_{t}^{A}(\mathbf{x}, \mathbf{y}), \mathbf{x}, \mathbf{y} \in \Omega_{A}$ by

$$
p_{t}^{A}(\mathbf{x}, \mathbf{y})=P_{\mathbf{x}}\left[B_{t} \in d \mathbf{y}, \sigma_{A}>t\right] /|d \mathbf{y}| .
$$

$p_{t}^{A}(\mathbf{x}, \mathbf{y})$ is symmetric and positive for all $\mathbf{x}, \mathbf{y} \in \Omega_{A}$ and $t>0$ and jointly continuous in $(t, \mathbf{x}, \mathbf{y}) \in(0, \infty) \times \Omega_{A}^{\circ} \times \Omega_{A}^{\circ}\left(\Omega_{A}^{\circ}\right.$ denotes the interior of $\left.\Omega_{A}\right)$. Readers may refer to [1] (Section 2.4, especially Theorem 4.4 and Proposition 4.9) for existence and properties of $p_{t}^{A}(\mathbf{x}, \mathbf{y})$. The set $A^{r}$ is Borel and $A \backslash A^{r}$ is polar, so that $P_{\mathbf{x}}\left[\sigma_{A}=\sigma_{A^{r}}\right]=1$ and $P_{\mathbf{x}}\left[B_{\sigma(A)} \in A^{r} \mid \sigma_{A}<\infty\right]=1$ for all $\mathbf{x}$ (see e.g. [1], [2], [3]). Denote by $\operatorname{nbd}_{\varepsilon}\left(A^{r}\right)$ the open $\varepsilon$-neighborhood of $A^{r}$ in $\mathbf{R}^{d}$. We also suppose

$$
\mathbf{R}^{d} \backslash \Omega_{A}=A^{r}
$$

( $A^{r}$ has no 'fine cavity'), which requires no essential change of the content of the paper since we are concerned only the exterior problem. Because of it we may write $\mathbf{x} \notin \operatorname{nbd}_{\varepsilon}\left(A^{r}\right)$ for $\mathbf{x} \in \Omega_{A} \backslash \operatorname{nbd}_{\varepsilon}\left(A^{r}\right)$. We write $x$ for the Euclidean length of $\mathbf{x} \in \mathbf{R}^{d}: x=|\mathbf{x}|$.

Let $d=2$ and $e_{A}(\mathbf{x})$ be the Green function for $\Omega_{A}$ with a pole at infinity normalized so that $e_{A}(\mathbf{x}) \sim \lg x$ when $x \rightarrow \infty$ (see (12) for a definition). The following theorem is stated only in the case $x \leq y$ which is not a restriction because $p_{t}^{A}(\mathbf{x}, \mathbf{y})$ is symmetric in $\mathbf{x}$ and $\mathbf{y}$ as mentioned above. 
Theorem 1. Let $d=2$. For any $M \geq 1$ and $\varepsilon>0$, uniformly for $\mathbf{x}, \mathbf{y} \notin \operatorname{nbd}{ }_{\varepsilon}\left(A^{r}\right)$ with $x \leq y<M t$, as $t \rightarrow \infty$

$$
\frac{p_{t}^{A}(\mathbf{x}, \mathbf{y})}{p_{t}^{(2)}(\mathbf{y}-\mathbf{x})} \sim \begin{cases}\frac{4 e_{A}(\mathbf{x}) e_{A}(\mathbf{y})}{(\lg t)^{2}} & \text { if } y \leq M \sqrt{t}, \\ \frac{e_{A}(\mathbf{x})}{\lg (t / y)} & \text { if } y>\sqrt{t}, x y \leq M t \text { and } y / t \rightarrow 0, \\ 1 & \text { if } x y \geq t / M \text { and } x \rightarrow \infty .\end{cases}
$$

The function $e_{A}$ agrees with $u$ appearing in (1) (verification is standard and given in [4]), so that Theorem 1 improves (1) by extending the range of validity to the regime $x \vee y<M \sqrt{n}$. The condition $\mathbf{x}, \mathbf{y} \notin \operatorname{nbd}_{\varepsilon}\left(A^{r}\right)$ can be relaxed to $\mathbf{x}, \mathbf{y} \in \Omega_{A}$ under some regularity condition for $\partial A$ (smoothness is sufficient: cf. Theorem 3 in Section 2.1 and a comment right after it), but not in general.

The case when $y>\delta t$ and $x<1 / \delta$ for a constant $\delta>0$ is excluded from the condition $x \leq y<M t$ in Theorem 1. In this case relation (44) breaks down (with the condition $y / t \rightarrow 0$ violated in the second case or the condition $x \rightarrow \infty$ in the third): in fact the second and third relations in (4) are complemented by the following result: there is a continuous function $c^{A}(\mathbf{x} ; \mathbf{v})$ taking values in $(0,1)$ such that under the constraints $y \asymp t$ and $x \asymp 1$, as $t \rightarrow \infty$

$$
p_{t}^{A}(\mathbf{x}, \mathbf{y}) \sim c^{A}(\mathbf{x} ; \mathbf{y} / t) p_{t}^{(2)}(\mathbf{y}-\mathbf{x})
$$

(Proposition 2.3), which combined with Theorem 1 shows that if $\mathbf{x}, \mathbf{y} \notin \operatorname{nbd}_{\varepsilon}\left(A^{r}\right)$ (for an $\varepsilon>0)$ and if

$$
x \vee y<M t \quad \text { and } \quad 1<t<M(x+1)(y+1)
$$

for a constant $M \geq 1$, then

$$
p_{t}^{A}(\mathbf{x}, \mathbf{y}) \asymp p_{t}^{(2)}(\mathbf{y}-\mathbf{x}) .
$$

Here the expression $f(t) \asymp g(t)$ means that the ratio $f(t) / g(t)$ is bounded away from both zero and infinity (provided that $f(x) g(x)>0$ ). Relation (5) is a lower bound since the upper bound is trivial and virtually follows if we show it with $A$ replaced by a disc $U(a)$ that includes $A$.

Let $\mathbf{e}=(1,0)$, the first coordinate vector of $\mathbf{R}^{2}$. The next theorem provides upper and lower bounds when $A=U(a)$ and $x \vee y \gg t$. (Here and in the sequel the expression $a \ll b$ $(a>0)$, used in informal expository passages, means that $b / a$ is large enough.) We have only to consider $\mathbf{y}=-y \mathbf{e}$. Write $\mathbf{x}=\left(x_{1}, x_{2}\right)$. It being readily shown (see proof of Lemma 3.7) that if $A \subset \overline{U(a)}$, then (5) is true under the condition

$$
a^{2}<t<\delta a y, x>(1+\delta) a \text { (for some } \delta>0 \text { ) and either } x_{1} \leq 0 \text { or }\left|x_{2}\right| \geq a
$$

we concentrate on the case when $x_{1}>0$ and $\left|x_{2}\right|<a$. For $x_{1}>0$, define the function $\rho=\rho(\mathbf{x}, \mathbf{y})$ by

$$
\rho=\frac{x_{1}}{x_{1}+y}
$$

Theorem 2. Let $d=2$. Suppose $t>a^{2}, x \leq y, \mathbf{y}=-y \mathbf{e}, x_{1}>0$ and $0 \leq x_{2}<a$.

(i) For each $\delta>0$, (5) holds if $A \subset \overline{U(a)}$, $\mathbf{x} \notin U(a(1+\delta))$ and

$$
\rho t \geq \delta a^{2} \wedge\left(a-x_{2}\right)^{2}
$$


(ii) For any $\delta>0$ there exist a constant $\kappa_{\delta}$ depending only on $\delta$ and a universal constant $c>0$ such that if $\mathbf{x} \notin U(a+\delta \sqrt{\rho t})$ and $a-x_{2} \geq \delta \sqrt{\rho t}$, then

$$
\frac{\kappa_{\delta} \sqrt{\rho t}}{a-x_{2}} \exp \left\{-k_{*}(\mathbf{x})\left(\frac{a-x_{2}}{\sqrt{\rho t}}+\delta\right)^{2}\right\} \leq \frac{p_{t}^{U(a)}(\mathbf{x}, \mathbf{y})}{p_{t}^{(2)}(\mathbf{x}-\mathbf{y})} \leq \frac{c \sqrt{\rho t}}{a-x_{2}} \exp \left\{-k(\mathbf{x}) \frac{\left(a-x_{2}\right)^{2}}{\rho t}\right\}
$$

with some functions $k_{*}(\mathbf{x})$ and $k(\mathbf{x})$ that satisfy

$$
\sqrt{2}-1<k(\mathbf{x})<\frac{1}{2}<k_{*}(\mathbf{x})<1 \quad \text { and } \quad \lim _{x_{1} \rightarrow \infty}\left(\frac{1}{2}-k(\mathbf{x})\right) x_{1}^{2}=\lim _{x_{1} \rightarrow \infty}\left(k_{*}(\mathbf{x})-\frac{1}{2}\right) x_{1}^{2}<\infty .
$$

(iii) If $x_{1}>2 a$ and $a^{2}<t<a y$, then

$$
\frac{p_{t}^{U(a)}(\mathbf{x}, \mathbf{y})}{p_{t}^{(2)}(\mathbf{x}-\mathbf{y})} \asymp\left[\frac{\sqrt{\rho t}}{a-x_{2}} \wedge 1\right] \exp \left\{-\left(\frac{1}{2}+O\left(a / x_{1}\right)\right) \frac{\left(a-x_{2}\right)^{2}}{\rho t}\right\}
$$

where the constants involved in $\asymp$ as well as in the $O$ term are universal.

In the case when $\left(a-x_{2}\right)^{2} / \rho t \rightarrow \infty$ and $a / x_{1} \rightarrow 0,(2 \pi)^{-1 / 2}$ times the right side of the formula in (iii) above may be heuristically regarded as an asymptotic form of the probability that a Brownian bridge joining $\mathbf{x}$ and $\mathbf{y}$ with length $t$ crosses the vertical axis at a level higher than $a$, for on the one hand if $B_{t}^{\prime}$ designates the second component of $B_{t}$,

$$
\sqrt{2 \pi} P_{\mathbf{x}}\left[B_{\rho t}^{\prime}>a\right] \sim \frac{\sqrt{\rho t}}{a-x_{2}} e^{-\left(a-x_{2}\right)^{2} / 2 \rho t}
$$

and on the other hand the distribution of the crossing time almost concentrates at $\rho t$.

Higher dimensional analogues of Theorems 1 and 2 will be given in Sections 2.4 and 3.3, respectively. The proofs are almost the same as to the two dimensional results. However, the formula corresponding to (6) is not similar to it and the result corresponding to Theorem 2 is accordingly modified.

REMARK 1. In the regime $x \vee y=O(t)$ the asymptotic behavior of $p_{t}^{A}(\mathbf{x}, \mathbf{y})$ is almost the same as that of $p_{t}^{U(a)}(\mathbf{x}, \mathbf{y})$ if $x \wedge y$ is large enough so that $e_{A}(\mathbf{x})$ and $e_{A}(\mathbf{y})$ are well approximated by $\lg x$ and $\lg y$, respectively. The situation becomes quite different when $(x \vee y) / t \rightarrow \infty$ : e.g., if the length of $\operatorname{pr}_{\mathbf{e}} A$ (the projection to the line perpendicular to $\mathbf{e}$ ) is zero and $\partial A$ is smooth, then $p_{t}^{A}(\mathbf{x}, y \mathbf{e}) \sim p_{t}^{(2)}(y \mathbf{e}-\mathbf{x})$ as $y / t \rightarrow \infty$ for all $\mathbf{x} \in \Omega_{A} \backslash \operatorname{nbd}_{\varepsilon}\left(A^{r}\right)$ (cf. [12, Theorem 3.5]).

In [4] Collet, Martinez and Martin employ a Harnack inequality and a classical expression of $p_{t}^{U(1)}$ as given in [5] to obtain an upper bound of $p_{t}^{A}$ and then use a compactness argument. The limiting function is identified by the uniqueness of the harmonic function $u$.

Our approach is quite different. If restricted to the first case of (44), our proof is based on the upper bounds

$$
\frac{P_{\mathbf{x}}\left[\sigma_{U(1)} \in d t, B_{t} \in d \xi\right]}{d t|d \xi|} \leq \frac{C}{t \lg t} \quad(1<x<\sqrt{t}, d \xi \subset \partial U(1))
$$

where $|d \xi|$ designates the length of $d \xi$, and

$$
P_{\mathbf{x}}\left[\sigma_{U(1)}<t\right]=o(1) \quad \text { if (and only if) } \quad \liminf \frac{\lg x}{\lg t} \geq \frac{1}{2}
$$


(as $t, x \rightarrow \infty)$ as well as on the following relation: for each $\varepsilon>0$, uniformly for $\mathbf{x} \in$ $U(r) \backslash \operatorname{nbd}_{\varepsilon}\left(A^{r}\right)$,

$$
P_{\mathbf{x}}\left[\sigma_{\partial U(r)}<\sigma_{A}\right] \sim \frac{e_{A}(\mathbf{x})}{\lg r} \quad \text { as } \quad r \rightarrow \infty .
$$

Relations (7), (8) and (9) are taken from [9], [11] and [12, respectively. Roughly speaking we first obtain (44), by using (17) and (8)), under the condition $x \sim y \sim \sqrt{t} / \lg t$ (a special case of the first one of (4)). This means that the Brownian bridge that connects $\mathbf{x}$ and $\mathbf{y}$ visits $A$ with so small a probability that $p_{t}^{A}(\mathbf{x}, \mathbf{y}) \sim p_{t}^{(2)}(0)$ in this special case. Next we consider the case $x<y \sim \sqrt{t} / \lg t$ and the event that the process starting at $\mathbf{x}$ escapes from $A$ before exiting the disc $U(\sqrt{t} / \lg t)$ with the exiting time $o(t)$. Formula (9) says that the probability of this escape from $A$ is asymptotic to $e_{A}(\mathbf{x}) / \lg \sqrt{t}$, which together with the preceding result shows $p_{t}^{A}(\mathbf{x}, \mathbf{y}) \sim 2(\lg t)^{-1} e_{A}(\mathbf{x}) p_{t}^{(2)}(0)$. For the case $x \vee y \ll \sqrt{t} / \lg t$, we have only to apply the latter case result to the Brownian motion started afresh at the exit time from the disc $U(\sqrt{t} / \lg t)$. These are to verify (44) in the case $x \leq y \leq C \sqrt{t} / \lg t$.

The proofs for the other cases of (4) need some results from [11] and [12] in addition to those mentioned above. The same method as described above applies to random walks as is discussed in [13].

The ratio on the left side of (4),$p_{t}^{A}(\mathbf{x}, \mathbf{y}) / p_{t}^{(d)}(\mathbf{y}-\mathbf{x})$, is the probability that the Brownian bridge joining $\mathbf{x}$ and $\mathbf{y}$ in the time interval $[0, t]$ avoids $A$, namely

$$
P_{\mathbf{x}}\left[B_{s} \notin A \text { for } 0 \leq s \leq t \mid B_{t}=\mathbf{y}\right]
$$

Thus formula (40) may be considered as giving the asymptotic form of this probability. In view of Theorem A.2 of Section 4 we have $P_{\mathbf{x}}\left[\sigma_{A}>t\right] \sim 2 e_{A}(\mathbf{x}) / \lg t$ if $\lim \sup \frac{\lg x}{\lg t} \leq \frac{1}{2}$. If $\lim \sup \frac{\lg (x \vee y)}{\lg t} \leq \frac{1}{2}$, the right side of (44) is therefore asymptotic to

$$
P_{\mathbf{x}}\left[\sigma_{A}>t\right] P_{\mathbf{y}}\left[\sigma_{A}>t\right]
$$

the probability that two independent Brownian motions starting at $\mathbf{x}$ and $\mathbf{y}$ both avoid $A$ until the time $t$. Collet et al. 4] points out (for their case) that (11) is interpreted as stating the coincidence of the asymptotic forms of these two probabilities for $\mathbf{x}$ and $\mathbf{y}$ restricted to a compact set of $\Omega_{A}$. Formula (4) shows that this coincidence extends over the regime in which $\lim \sup \frac{\lg (x \vee y)}{\lg t} \leq \frac{1}{2}$ but not necessarily beyond it. It may be worth noting that since the law of the Brownian Bridge is not affected by a constant drift, for any constant vector $\mathbf{v}$ the transition density of the process $B_{s}^{\mathbf{v}}:=B_{s}+\mathbf{v} s$ killed upon hitting $A$ is given by

$$
\frac{p_{t}^{A}(\mathbf{x}, \mathbf{y})}{p_{t}^{(d)}(\mathbf{y}-\mathbf{x})} p_{t}^{(d)}(\mathbf{y}-\mathbf{v} t-\mathbf{x})
$$

hence Theorem 1 also determines its asymptotic form.

We separate Theorem 1 into three propositions (Theorem 3, Proposition 2.1 and 2.2) corresponding to the three cases in (4), namely cases (1) $x \vee y \leq \sqrt{t},(2) x y \leq t, x \vee y>\sqrt{t}$ and (3) $x y>t$, and deal with them in Sections 2.1, 2.2 and 2.3, respectively. The result corresponding to Theorem 1 in the higher dimensions $d \geq 3$ will be briefly discussed in Section 2.4, the proof being much simpler. Theorem 2 is proved in Section 3. The higher dimensional result for it is given in Section 3.4; the result itself somewhat differs from the two dimensional one although its proof is similar. In Section 4 we collect the results from [9], [10], [11] and [12] that are applied in this paper. 


\section{Asymptotic forms in the regime $x \vee y=O(t)$}

For $\mathbf{x} \in \Omega_{A}$, define

$$
H_{A}(\mathbf{x}, t ; d \xi)=\frac{P_{\mathbf{x}}\left[B_{\sigma_{A}} \in d \xi, \sigma_{A} \in d t\right]}{d t} \quad(d \xi \subset \partial A, t>0),
$$

the hitting distribution of $A$ in space-time, which in addition to the things introduced in Section 1 will be of fundamental use throughout the rest of the paper. We shall apply some asymptotic estimates of $H_{A}$ (or rather its partial integrals) that are taken from [9], [10], [11] and [12. We collect them in Section 4 for convenience of citation.

Let $d=2$. The function $e_{A}(\mathbf{x}), \mathbf{x} \in \Omega_{A}$ may be defined by

$$
e_{A}(\mathbf{x})=\pi \lim _{|\mathbf{y}| \rightarrow \infty} g_{\Omega_{A}}(\mathbf{x}, \mathbf{y})
$$

where $g_{\Omega_{A}}(\mathbf{x}, \mathbf{y})=\int_{0}^{\infty} p_{t}^{A}(\mathbf{x}, \mathbf{y}) d t\left(\mathbf{x}, \mathbf{y} \in \Omega_{A}\right)$, the Green function for the set $\Omega_{A}$; in particular $e_{A}$ is harmonic in the interior of $\Omega_{A}$. Let $\operatorname{err}_{*}(\mathbf{x}, t)$ stand for any function satisfying that $\operatorname{err}_{*}(\mathbf{x}, t)=0$ if $x:=|\mathbf{x}| \geq 2 R_{A}$ and

$$
\left|\operatorname{err}_{*}(\mathbf{x}, t)\right| \leq C P_{\mathbf{x}}\left[\sigma_{A \cup \partial U\left(2 R_{A}\right)}>t / \lg t\right] \quad \text { if } \quad x<2 R_{A} .
$$

It is noted that the right side above is dominated by $C e^{-\lambda t / R_{A}^{2} \lg t}$ with a certain universal constant $\lambda>0(*$ is attached to distinguish from the notation $\operatorname{err}(\mathbf{x}, t)$ used in [12]).

We often write $p_{t}^{(d)}(\mathbf{x})$ for $p_{t}^{(d)}(x)$ for convenience sake; also write $\sigma(A)$ for $\sigma_{A}$ for typographical reason; $x \vee y$ and $x \wedge y$ denote the maximum and minimum of real numbers $x, y$, respectively.

\subsection{Case $x \vee y=O(\sqrt{t}), d=2$}

In this and the next subsections we assume $d=2$ and simply write $p_{t}(x)$ for $p_{t}^{(2)}(x)$ (and also $p_{t}(\mathbf{x})$ for $\left.p_{t}^{(2)}(\mathbf{x})\right)$. The following theorem refines the first case of (4).

Theorem 3. For any $M>1$, uniformly for $\mathbf{x}, \mathbf{y} \in \Omega_{A}$ subject to the constraint $x \vee y<$ $M \sqrt{t}$, as $t \rightarrow \infty$

$$
p_{t}^{A}(\mathbf{x}, \mathbf{y})=\frac{4 e_{A}(\mathbf{x}) e_{A}(\mathbf{y})}{(\lg t)^{2}} p_{t}(\mathbf{y}-\mathbf{x})\left(1+O\left(\frac{\lg \lg t}{\lg t}\right)\right)+\operatorname{err}_{*}(\mathbf{x}, t)+\operatorname{err}_{*}(\mathbf{y}, t)
$$

The constants involved in the $O$ term appearing in (13) of course depends on $M$ and the same remark applies to the results that follow. If the boundary $\partial A$ is smooth, then $\operatorname{err}_{*}(\mathbf{x}, t) \leq C e_{A}(\mathbf{x}) e^{-\lambda \sqrt{t} / R_{A}^{2} \lg t}$ so that $\operatorname{err}_{*}(\mathbf{x}, t)+\operatorname{err}_{*}(\mathbf{y}, t)$ may be deleted from the right side of (13) unless $\mathbf{x}$ and $\mathbf{y}$ approach $\partial A$ simultaneously.

The proof of Theorem 3 will be given at the end of this subsection after showing Lemmas 2.1 and 2.2 given below. Lemmas 2.1 and 2.2 improve the first relation of (4) by giving error estimates and extending the range of validity of the formula beyond the restriction $x \vee y=O(\sqrt{t})$. In order to include the improvement we shall apply certain refined versions of (7), (8) and (9); otherwise we do not need such refined ones. We suppose $d=2$ throughout this and the next subsections.

Lemma 2.1. For any $M \geq 1$, as $x \wedge y \wedge t \rightarrow \infty$ under the constraints $\lg x \sim \lg y \sim \frac{1}{2} \lg t$ and $x y \leq M t$,

$$
p_{t}^{A}(\mathbf{x}, \mathbf{y})=p_{t}(\mathbf{y}-\mathbf{x})\left[1+O\left(\frac{1}{\lg t}\left[1 \vee \lg \frac{t}{x^{2} \wedge y^{2}}\right]\right)\right]
$$


Proof. Since $p_{t}^{A}(\mathbf{x}, \mathbf{y}) \leq p_{t}^{U\left(R_{A}\right)}(\mathbf{x}, \mathbf{y}) \leq p_{t}(\mathbf{y}-\mathbf{x})$, it suffices to obtain a proper upper bound of the difference $p_{t}(\mathbf{y}-\mathbf{x})-p_{t}^{U\left(R_{A}\right)}(\mathbf{x}, \mathbf{y})$. To this end we use the identity

$$
p_{t}(\mathbf{y}-\mathbf{x})-p_{t}^{U\left(R_{A}\right)}(\mathbf{x}, \mathbf{y})=\int_{0}^{t} d s \int_{\partial U\left(R_{A}\right)} H_{U\left(R_{A}\right)}(\mathbf{x}, s ; d \xi) p_{t-s}(\mathbf{y}-\xi) .
$$

Let $I_{[a, b]}$ denote the double integral in (14) restricted to $[a, b] \times \partial A, 0 \leq a<b \leq t$. Suppose $R_{A}=1$ for simplicity. Let $x y<M t$. We may suppose $x \leq y$, so that $x<\sqrt{M t}$. On noting $p_{t-s}(\mathbf{y}-\xi) \leq C p_{t}(y)$ for $\xi \in \partial U(1), s<t / 2$,

$$
I_{[0, t / 2]} \leq C p_{t}(y) P_{\mathbf{x}}\left[\sigma_{U(1)} \leq t / 2\right] .
$$

We deduce from Theorem A.2 of Section 4 (with $U(1)$ in place of $A$ ) that if $\lg x \sim \frac{1}{2} \lg t$

$$
P_{\mathbf{x}}\left[\sigma_{U(1)} \leq t / 2\right] \leq \frac{C\left[1 \vee \lg \left(t / x^{2}\right)\right]}{\lg t}
$$

we also have $p_{t}(y) \leq p_{t}(\mathbf{y}-\mathbf{x}) e^{2 M}$. Putting these together we obtain

$$
I_{[0, t / 2]} \leq C e^{2 M} \frac{1 \vee \lg \left(t / x^{2}\right)}{\lg t} p_{t}(\mathbf{y}-\mathbf{x})
$$

For the other part $I_{[t / 2, t]}$ we apply Theorems A.1 and A.4 to see that if $\lg x \sim \lg y \sim \frac{1}{2} \lg t$, then

$$
I_{[t / 2, t]} \leq \frac{C p_{t}(x)}{\lg t} \int_{\partial U(1)}|d \xi| \int_{t / 2}^{t} p_{t-s}(\mathbf{y}-\xi) d s .
$$

Writing $r$ for $|\mathbf{y}-\xi|$ and changing the variable by $u=r^{2} / 2(t-s)$ so that $d s /(t-s)=d u / u$, we compute the inner integral to obtain an upper bound of it as follows:

$$
\int_{t / 2}^{t} p_{t-s}(r) d s=\frac{1}{2 \pi} \int_{r^{2} / t}^{\infty} \frac{e^{-u}}{u} d u \leq C\left[e^{-r^{2} / t} \vee \lg \frac{t}{r^{2}}\right] \leq C^{\prime} e^{-r^{2} / t}\left[1 \vee \lg \frac{t}{r^{2}}\right]
$$

and then infer that

$$
I_{[t / 2, t]} \leq C e^{M} p_{t}(\mathbf{y}-\mathbf{x}) \frac{1 \vee \lg \left(t / y^{2}\right)}{\lg t}
$$

Combined with (15) this concludes the proof of the lemma.

Lemma 2.2. For $M \geq 1$, if $\mathbf{x} \in \Omega_{A}, x<M \sqrt{t} / \lg t, y<M \sqrt{t}$ and $\lg y \sim \frac{1}{2} \lg t$, then

$$
p_{t}^{A}(\mathbf{x}, \mathbf{y})=\frac{2 e_{A}(\mathbf{x})+\operatorname{err}_{*}(\mathbf{x}, t)}{\lg t} p_{t}(y)(1+o(1))
$$

moreover if $y \geq \sqrt{t} /(\lg t)^{\delta}$ for some $\delta>0$ in addition, the error term o(1) above can be replaced by

$$
O((\lg \lg t) / \lg t)
$$

Proof. Let $x<r:=M \sqrt{t} / \lg t, y<M \sqrt{t}$ and $T:=2 t / \lg t$ and decompose

$$
\begin{aligned}
p_{t}^{A}(\mathbf{x}, \mathbf{y})= & \iint_{[0, T] \times \partial U(r)} P_{\mathbf{x}}\left[\sigma_{\partial U(r)} \in d s, \sigma_{A}>s, B_{\sigma_{\partial U(r)}} \in d \xi\right] p_{t-s}^{A}(\xi, \mathbf{y}) \\
& +\varepsilon(t, \mathbf{x}, \mathbf{y})
\end{aligned}
$$


where

$$
\varepsilon(t, \mathbf{x}, \mathbf{y})=\int_{U(r) \cap \Omega_{A}} P_{\mathbf{x}}\left[\sigma_{\partial U(r)} \wedge \sigma_{A}>T, B_{T} \in d \mathbf{z}\right] p_{t-T}^{A}(\mathbf{z}, \mathbf{y}) .
$$

It holds that

$$
P_{\mathbf{x}}\left[\sigma_{\partial U(r)} \wedge \sigma_{A}>T\right] \leq C e^{-\lambda T / r^{2}}\left(e_{A}(\mathbf{x})+P\left[\sigma_{\partial U\left(2 R_{A}\right)} \wedge \sigma_{A}>\frac{1}{2} T\right]\right)
$$

with some universal constant $\lambda>0$. Indeed, this is trivial if $x>R:=2 R_{A}$ for which $e_{A}(\mathbf{x})>\lg 2$ (cf.(68) $)$, whereas, by applying strong Markov property at the exit time $\sigma_{\partial U(R)} \wedge$ $\sigma_{A}=\sigma_{A \cup \partial U(R)}$ and breaking the event $\sigma_{\partial U(r)} \wedge \sigma_{A}>T$ according as $\sigma_{\partial U(R)}$ is less than $T / 2$ or not, we infer that for $\mathbf{x} \in U(R) \backslash A^{r}$,

$$
P_{\mathbf{x}}\left[\sigma_{\partial U(r)} \wedge \sigma_{A}>T\right] \leq\left(P_{\mathbf{x}}\left[\sigma_{\partial U(R)}<\sigma_{A}\right]+P_{\mathbf{x}}\left[\sigma_{A \cup \partial U(R)}>\frac{1}{2} T\right]\right) \sup _{\mathbf{y} \in U(R)} P_{\mathbf{y}}\left[\sigma_{\partial U(r)}>\frac{1}{2} T\right] .
$$

The supremum above is $O\left(e^{-\lambda T / r^{2}}\right)$; use Theorem A.3 to see $P_{\mathbf{x}}\left[\sigma_{\partial U(R)}<\sigma_{A}\right] \leq e_{A}(\mathbf{x}) / \lg 2$. Thus we have $(\underline{18})$.

By $y \leq M \sqrt{t}$ we have

$$
r y / t \leq M^{2} / \lg t
$$

Then, noting $p_{t-T}^{A}(\mathbf{z}, \mathbf{y}) \leq p_{t-T}(\mathbf{y}-\mathbf{z}) \leq c_{M} p_{t}(y)$ for $z \leq r$, we observe

$$
\varepsilon(t, \mathbf{x}, \mathbf{y}) \leq c_{M}^{\prime} e^{-\lambda T / r^{2}}\left[e_{A}(\mathbf{x})+\operatorname{err}_{*}(\mathbf{x}, t)\right] p_{t}(y)
$$

hence $\varepsilon(t, \mathbf{x}, \mathbf{y})$ is absorbed into the error terms since $T / r^{2} \geq M^{-2} \lg t$.

For $s<T, \xi \in \partial U(r)$, by Lemma 2.1 we have

$$
p_{t-s}^{A}(\xi, \mathbf{y})=p_{t-s}(\mathbf{y}-\xi)(1+o(1)) ;
$$

observing $T|\mathbf{y}-\xi|^{2} / t^{2} \leq 2 M^{2} / \lg t$, from (19) we deduce

$$
p_{t-s}(\mathbf{y}-\xi)=p_{t}(y)\left(1+O\left(\frac{1}{\lg t}\right)\right) .
$$

On using Theorem A.3 of Section 4 as well as (18)

$$
\begin{aligned}
P_{\mathbf{x}}\left[\sigma_{\partial U(r)}<T \wedge \sigma_{A}\right] & =P_{\mathbf{x}}\left[\sigma_{\partial U(r)}<\sigma_{A}\right]-P_{\mathbf{x}}\left[T<\sigma_{\partial U(r)}<\sigma_{A}\right] \\
& =\frac{e_{A}(\mathbf{x})+\operatorname{err}_{*}(\mathbf{x}, t)}{\lg r}\left(1+O\left(\frac{1}{\lg r}\right)\right) .
\end{aligned}
$$

Now the double integral in (17) may be written as

$$
P_{\mathbf{x}}\left[\sigma_{\partial U(r)}<T \wedge \sigma_{A}\right] p_{t}(y)(1+o(1))=\frac{e_{A}(\mathbf{x})+\operatorname{err}_{*}(\mathbf{x}, t)}{\lg r} p_{t}(y)(1+o(1)) .
$$

Since $\lg r=\frac{1}{2} \lg t+O(\lg \lg t)$, this verifies the first half of the lemma.

If $y \geq \sqrt{t} /(\lg t)^{\delta}$, then in (21) $o(1)$ can be replaced by $O((\lg \lg t) / \lg t)$ according to Lemma 2.1 (note by (19) we also have $|\mathbf{y} \cdot \xi| / t \leq r y / t=O(1 / \lg t)$ ). This permits replacing $o(1)$ by $O((\lg \lg t) / \lg t)$ in (24), and thereby yields the required error bound.

Proof of Theorem [3. The case $y \geq x \geq \sqrt{t} / \lg t$ is covered by Lemma 2.1 (see (68) in Section 4 for the error arising from the replacement of $e_{A}(\mathbf{x})$ by $\left.\lg x\right)$. First suppose $x \leq y \leq M \sqrt{t} / \lg t$. Then, by Lemma 2.2 we see in the double integral in (17)

$$
p_{t-s}^{A}(\xi, \mathbf{y})=p_{t-s}^{A}(\mathbf{y}, \xi)=\frac{2 e_{A}(\mathbf{y})+\operatorname{err}_{*}(\mathbf{y}, t)}{\lg t} p_{t-s}(\xi)\left(1+O\left(\frac{\lg \lg t}{\lg t}\right)\right) .
$$


Since $p_{t-s}(\xi)=p_{t}(0)(1+O(1 / \lg t))$ for $s \leq T$, we may write (17) as

$$
p_{t}^{A}(\mathbf{x}, \mathbf{y})=P_{\mathbf{x}}\left[\sigma_{\partial U(r)}<T \wedge \sigma_{A}\right] \frac{2 e_{A}(\mathbf{y})+\operatorname{err}_{*}(\mathbf{y}, t)}{\lg t} p_{t}(0)\left(1+O\left(\frac{\lg \lg t}{\lg t}\right)\right)+\varepsilon(t, \mathbf{x}, \mathbf{y}) .
$$

The evaluation of the term $\varepsilon(t, \mathbf{x}, \mathbf{y})$ given in (20) and that of $P_{\mathbf{x}}\left[\sigma_{\partial U(r)}<T \wedge \sigma_{A}\right]$ in (23) are valid. Thus we conclude the required relation of the Theorem 3 . Finally in the case $x \leq \sqrt{t} / \lg t \leq y$, we have $x y \leq M t / \lg t$, so that $p_{t}(y)=p_{t}(\mathbf{y}-\mathbf{x})+O(1 / \lg t)$ and applying the second half of Lemma 2.2 finishes the proof.

\subsection{Case $x y=O(t)$ and $x \vee y>\sqrt{t}, d=2$}

We continue to suppose $d=2$ and write $p_{t}(x)$ for $p_{t}^{(2)}(x)$ as mentioned previously.

Proposition 2.1. Let $d=2$. For each $M \geq R_{A}$ and $\varepsilon>0$, uniformly for $\mathbf{x} \notin \operatorname{nbd}_{\varepsilon}\left(A^{r}\right)$ and $\mathbf{y}$ subject to the constraints $y>\sqrt{t}$ and $x y<M t$, as $y / t \rightarrow 0$ and $t \rightarrow \infty$

$$
p_{t}^{A}(\mathbf{x}, \mathbf{y})=\frac{e_{A}(\mathbf{x})}{\lg (t / y)} p_{t}(\mathbf{y}-\mathbf{x})(1+o(1))
$$

Proof. The case when $y / \sqrt{t}$ is bounded is covered by Lemmas 2.1 and 2.2 in conjunction. Hence we suppose that $y / \sqrt{t} \rightarrow \infty$ as well as $y / t \rightarrow 0$ in addition to the constraint put in the lemma. From $x y<M t$ it then follows that $x / \sqrt{t} \rightarrow 0$ and $x \rightarrow \infty$.

Identity (14) is valid with $A$ replacing $U\left(R_{A}\right)$, namely

$$
p_{t}(\mathbf{y}-\mathbf{x})-p_{t}^{A}(\mathbf{x}, \mathbf{y})=\int_{0}^{t} d s \int_{\partial A} H_{A}(\mathbf{x}, s ; d \xi) p_{t-s}(\mathbf{y}-\xi)
$$

We are to compute the integral on the right side to find the asymptotic form of $p_{t}(\mathbf{y}-$ $\mathbf{x})-p_{t}^{A}(\mathbf{x}, \mathbf{y})$. Since $P_{\mathbf{x}}\left[\sigma_{A}>t / 2\right] \leq C e_{A}(\mathbf{x}) / \lg t$ (see (29) below) and $p_{t-s}(\mathbf{y}-\xi), \xi \in \partial A$ is $O\left(p_{t / 2}(y)\right)$ for $s>t / 2$, the integral restricted to $[t / 2, t]$ is negligible. Because of the condition $y / t \rightarrow 0, p_{t-s}(\mathbf{y}-\xi)$ may be replaced by $p_{t-s}(y)$. It therefore suffices to identify the asymptotic form of

$$
\int_{0}^{t / 2} p_{t-s}(y) P_{\mathbf{x}}\left[\sigma_{A} \in d s\right]
$$

We shall write $J_{[a, b]}$ for this integral restricted to an interval $[a, b], 0 \leq a<b \leq t / 2$. Put

$$
\eta=(t / y)^{2}
$$

Then $\eta \rightarrow \infty$ and $\eta / t \rightarrow 0$ in our present setting. Expanding $1 /(t-s)$ into the Taylor series of $s / t$ yields

$$
p_{t-s}(y)=\frac{1}{1-\frac{s}{t}} p_{t}(y) \exp \left\{-\frac{s}{2 \eta}\left(1+\frac{s}{t}+\frac{s^{2}}{t^{2}}+\cdots\right)\right\} .
$$

Taking constants $\alpha>1$ large and $0<\delta<1$ small we split the integral at $\delta \eta$ and $\alpha \eta$ and observe

$$
\begin{gathered}
J_{[0, \delta \eta]}=\int_{0}^{\delta \eta} p_{t-s}(y) P_{\mathbf{x}}\left[\sigma_{A} \in d s\right]=p_{t}(y) P_{\mathbf{x}}\left[\sigma_{A}<\delta \eta\right](1+O(\delta)) \\
J_{[\alpha \eta, t / 2]} \leq 2 p_{t}(y) \int_{\alpha \eta}^{\infty} e^{-s / 2 \eta} P_{\mathbf{x}}\left[\sigma_{A} \in d s\right] \leq 2 p_{t}(y) P_{\mathbf{x}}\left[\sigma_{A}>\alpha \eta\right] e^{-\alpha / 2}
\end{gathered}
$$


and

$$
J_{[\delta \eta, \alpha \eta]} \leq 2 p_{t}(y) P_{\mathbf{x}}\left[\delta \eta<\sigma_{A}<\alpha \eta\right] .
$$

Let $\mathbf{x} \notin \operatorname{nbd}_{\varepsilon}\left(A^{r}\right)$. Then Theorem A.2 entails that for each $\alpha^{\prime}>0$, uniformly for $s>\alpha^{\prime} x^{2}$

$$
P_{\mathbf{x}}\left[\sigma_{A}>s\right]=\frac{2 e_{A}(\mathbf{x})}{\lg s}\left[1+O\left(\frac{1}{\lg s}\right)\right] .
$$

Note that the condition $x y<M t$ is written as $x^{2}<M^{2} \eta$ and then deduce

$$
\begin{gathered}
\frac{J_{[0, \delta \eta]}}{p_{t}(y)}=P_{\mathbf{x}}\left[\sigma_{A}<\delta \eta\right](1+O(\delta))=\left(1-\frac{2 e_{A}(\mathbf{x})}{\lg (\delta \eta)}(1+o(1))\right)(1+O(\delta)), \\
\frac{J_{[\alpha \eta, t / 2]}}{p_{t}(y)} \leq 2 e^{-\alpha / 2} \frac{e_{A}(\mathbf{x})}{\lg (\alpha \eta)}(1+o(1)) \quad \text { and } \quad \frac{J_{[\delta \eta, \alpha \eta]}}{p_{t}(y)} \leq \frac{4 e_{A}(\mathbf{x})}{(\lg \eta)^{2}}[\lg (\alpha / \delta)+O(1)] .
\end{gathered}
$$

Then, since $1 / \delta$ and $\alpha$ may be arbitrarily large, we find that

$$
J_{[0, t / 2]}=\left(1-\frac{2 e_{A}(\mathbf{x})}{\lg \eta}(1+o(1))\right) p_{t}(y) .
$$

Remembering that the left side equals $p_{t}(\mathbf{y}-\mathbf{x})-p_{t}^{A}(\mathbf{x}, \mathbf{y})-J_{[t / 2, t]}$ and $J_{[t / 2, t]}$ is negligible, we conclude (25), for if $x^{2} / \eta \rightarrow 0$ (namely, $x y / t \rightarrow 0$ ), then $p_{t}(y) \sim p_{t}(\mathbf{y}-\mathbf{x})$, whereas if $x^{2}>\alpha^{\prime} \eta$ for some constant $\alpha^{\prime}>0$, then $2 e_{A}(\mathbf{x}) / \lg \eta \rightarrow 1$, so that $J_{[0, t / 2]}=o\left(p_{t}(y)\right)=$ $o\left(p_{t}(\mathbf{y}-\mathbf{x})\right)$. The proof of Proposition 2.1 is complete.

\subsection{Case $x y>t / M, d \geq 2$}

Proposition 2.2. Let $d \geq 2$. For each $M \geq 1$, uniformly for $\mathbf{x}$ and $\mathbf{y}$ subject to the constraint $x y>t / M$ and $x \vee y<M t$, as $x \wedge y \rightarrow \infty$

$$
p_{t}^{A}(\mathbf{x}, \mathbf{y})=p_{t}^{(d)}(\mathbf{y}-\mathbf{x})(1+o(1))
$$

For the proof we shall use the first half of the following

Lemma 2.3. Let $d \geq 1$ and $\nu=\frac{1}{2} d-1$. For any $\delta>0$ there exists a constant $C_{\delta}$ that depends only on $\delta$ and $d$ such that if $(x+z) z>\delta t>0$, then

(i) for $0<\theta<1$

$$
\theta^{d / 2} \int_{\theta t}^{t} p_{s}^{(d)}(x) p_{t-s}^{(d)}(z) d s \leq C_{\delta} p_{t}^{(d)}(x+z)\left(\frac{x+z}{t z}\right)^{\nu} \sqrt{\frac{t}{(x+z) z}}
$$

(ii) if $z \leq x$ in addition, $\int_{0}^{t} p_{s}^{(d)}(x) p_{t-s}^{(d)}(z) d s$ is dominated by the right side above.

Proof. The computation is based on the identity

$$
p_{s}^{(d)}(\mathbf{x}) p_{t-s}^{(d)}(\mathbf{z})=p_{t}^{(d)}(\mathbf{z}-\mathbf{x}) p_{T}^{(d)}\left(\frac{t-s}{t} \mathbf{x}+\frac{s}{t} \mathbf{z}\right)
$$

where $T=(t-s) s / t$ (recall $p_{s}^{(d)}(\mathbf{x})$ is written for $\left.p_{t}^{(d)}(x)\right)$. Our task will be to evaluate the integral

$$
J:=\int_{\theta t}^{t} p_{T}^{(d)}\left(\frac{t-s}{t} \mathbf{x}+\frac{s}{t} \mathbf{z}\right) d s=\int_{\theta t}^{t} p_{T}^{(d)}\left(\frac{t-s}{t}(\mathbf{x}-\mathbf{z})+\mathbf{z}\right) d s
$$


from above. Choose the directions of $\mathbf{x}$ and $\mathbf{z}$ so that $\mathbf{z}$ is a negative multiple of $\mathbf{x}$; the value is unaltered by this choice, for so is the left side of (31). Then $p_{t}^{(d)}(\mathbf{z}-\mathbf{x})=p_{t}^{(d)}(x+z)$ and the integrand becomes $p_{T}^{(d)}\left(\frac{t-s}{t}(x+z)-z\right)$, so that after substitution of $t-s=u$

$$
J=\int_{0}^{(1-\theta) t} p_{T}^{(d)}\left(\frac{u}{t}(x+z)-z\right) d u, \quad T=\frac{u(t-u)}{t} .
$$

Write the identity $\int_{0}^{\infty} \exp \left\{-\frac{1}{2} \alpha^{2} u-\frac{1}{2} \beta^{2} u^{-1}\right\} u^{-\nu-1} d u=2(\alpha / \beta)^{\nu} K_{\nu}(\alpha \beta)$ (cf. [6], p.146) as

$$
\int_{0}^{\infty} p_{u}^{(d)}(\alpha u-\beta) d u=2(2 \pi)^{-d / 2}(\alpha / \beta)^{\nu} K_{\nu}(\alpha \beta) e^{\alpha \beta},
$$

where $\alpha \beta>0, \nu=\frac{1}{2} d-1$ and $K_{\nu}$ is the usual modified Bessel function of order $\nu$. Then, noting that $\theta u<T<u$ for $0<u<(1-\theta) t$, we have

$$
\begin{aligned}
J & \leq \frac{1}{\theta^{d / 2}} \int_{0}^{\infty} p_{u}^{(d)}\left(\frac{x+z}{t} u-z\right) d u \\
& =2\left(\frac{1}{2 \pi \theta}\right)^{d / 2}\left(\frac{x+z}{t z}\right)^{\nu} K_{\nu}\left(\frac{(x+z) z}{t}\right) e^{(x+z) z / t}
\end{aligned}
$$

Combined with the following asymptotic formula:

$$
K_{\nu}(\eta) e^{\eta} \sim \sqrt{\pi / 2 \eta} \quad \text { as } \quad \eta \rightarrow \infty
$$

(for every $\nu \in \mathbf{R}$ ) (cf. [7]) this yields (i).

Let $z \leq x$ and compare the function $|(t-s) x-s z|$ restricted on $0<s<t / 2$ with that on $t / 2<s<t$. Then by symmetry of $T=s(t-s) / t$ about $s=t / 2$ we see that the integral of $p_{T}^{(d)}\left(\frac{t-s}{t} x-\frac{s}{t} z\right) d s$ on $[t / 2, t]$ is not less than that on $[0, t / 2]$, so that (ii) follows from (i).

Proof of Proposition 2.2. We may suppose $A=U\left(R_{A}\right)$. Let $R_{A}=1$ and $1<y \leq$ $x<M t$. We evaluate the difference $p_{t}(\mathbf{y}-\mathbf{x})-p_{t}^{U(1)}(\mathbf{x}, \mathbf{y})$ by means of (14). As before the integral in (14) restricted on $[a, b] \times \partial U(1)$ is denoted by $I_{[a, b]}$.

Let $d=2$ and write $p_{t}(x)$ for $p_{t}^{(2)}(x)$. Then, using Theorems A.1 and A.4 (of Section 4) we have

$$
\begin{aligned}
I_{[t / 4, t]} & =\int_{t / 4}^{t} d s \int_{\partial U(1)} H_{U(1)}(\mathbf{x}, s ; d \xi) p_{t-s}(\mathbf{y}-\xi) \\
& \leq \frac{C e^{M}}{1 \vee \lg (t / x)} \int_{\partial U(1)}|d \xi| \int_{t / 4}^{t} p_{s}(\mathbf{x}) p_{t-s}(\mathbf{y}-\xi) d s
\end{aligned}
$$

Suppose $x y>t / M$ and apply Lemma 2.3 with $\mathbf{z}=\mathbf{y}-\xi$. Noting $p_{t}(\mathbf{y}-\xi-\mathbf{x}) \leq$ $C e^{2 M} p_{t}(\mathbf{y}-\mathbf{x})$ we then deduce the bound

$$
\frac{I_{[t / 4, t]}}{p_{t}(\mathbf{y}-\mathbf{x})} \leq \frac{c_{M}}{1 \vee \lg (t / x)} \sqrt{\frac{t}{x y}}
$$

and, by considering each case of $t / x$ being bounded from below or not, we infer that the right side above approaches zero as $t \rightarrow \infty$. 
For $s \in[0, t / 4]$ and $\xi \in \partial U(1)$, we have $p_{t-s}(\mathbf{y}-\xi) \leq C p_{t}(y)$, and using Theorem A.2 we deduce

$$
I_{[0, t / 4]} \leq C p_{t}(y) P_{\mathbf{x}}\left[\sigma_{U(1)}<t / 4\right] \leq c_{M} p_{t}(y) \frac{1}{1 \vee \lg (t / x)} \cdot \frac{t}{x^{2}} e^{-2 x^{2} / t}
$$

Since $x^{2} \geq t / M$ and $p_{t}(y) e^{-2 x^{2} / t}=e^{-y^{2} / 2 t} p_{t}(2 x) \leq e^{-x^{2} / 2 t} p_{t}(x+y)$, we obtain

$$
I_{[0, t / 4]} \leq \frac{M c_{M}}{1 \vee \lg (t / x)} e^{-x^{2} / 2 t} p_{t}(x+y)
$$

Thus $I_{[0, t / 4]}$ is negligible and for $d=2$, the assertion of the theorem follows.

For $d \geq 3$ we apply Theorem A.1 to see that $P_{\mathbf{x}}\left[\sigma_{U(1)}<t\right] \leq C x^{-2} t^{-\nu} p_{t}^{(d)}(x-1)$ if $x^{2} \geq t$ and the same arguments as above yield

$$
\frac{I_{[t / 4, t]}}{p_{t}^{(d)}(\mathbf{y}-\mathbf{x})} \leq \frac{c_{M}}{y^{\nu}}\left(\frac{x}{t}\right)^{\nu} \sqrt{\frac{t}{x y}} \text { and } \frac{I_{[0, t / 4]}}{p_{t}^{(d)}(x+y)} \leq \frac{c_{M}}{t^{\nu}} e^{-x^{2} / 2 t}
$$

in place of (34) and (35), respectively. The right sides tending to zero in both inequalities above, the theorem is thus proved.

\subsection{The higher dimensions I}

Let $d \geq 3$ and put

$$
u_{A}(\mathbf{x})=P_{\mathbf{x}}\left[\sigma_{A}=\infty\right]
$$

Theorem 4. Let $d \geq 3$. For each $\varepsilon>0$, uniformly for $\mathbf{x}, \mathbf{y} \notin \operatorname{nbd}_{\varepsilon}\left(A^{r}\right)$, as $t \rightarrow \infty$ and $(x \vee y) / t \rightarrow 0$

$$
p_{t}^{A}(\mathbf{x}, \mathbf{y})=u_{A}(\mathbf{x}) u_{A}(\mathbf{y}) p_{t}^{(d)}(\mathbf{y}-\mathbf{x})(1+o(1)) ;
$$

moreover for each $M \geq 1$, this equality still holds true as $x \wedge y \wedge t \rightarrow \infty$ under the constraint $M^{-1} t<x \vee y<M t$.

First suppose $x \wedge y \rightarrow \infty$. Then, under the additional restriction $x y>t$ the assertion of Theorem 4 follows from Proposition 2.2. The other case $x y \leq t$ is easily disposed of by examining the proofs of Lemmas 2.1 and 2.2. Indeed in the proof of Lemma 2.1 (assuming $x \geq y$ differently from therein) we obtain that if $1<y \leq \sqrt{t} \leq x \leq t$, then

$$
I_{[0, t / 2]} \leq C x^{-(d-2)} p_{t}^{(d)}(\mathbf{y}-\mathbf{x})
$$

in place of (15) since $P_{\mathbf{x}}\left[\sigma_{U(1)}<t / 2\right] \leq P_{\mathbf{x}}\left[\sigma_{U(1)}<\infty\right]=x^{-(d-2)}$ and

$$
I_{[t / 2, t]} \leq C y^{-(d-2)} p_{t}^{(d)}(\mathbf{y}-\mathbf{x})
$$

in place of (16) owing to Theorem A.1 (observe $\int_{0}^{t / 2} p_{s}^{(d)}(r) d s \leq \mathrm{Cr}^{-(d-2)}$ ). Thus

$$
p_{t}^{A}(\mathbf{x}, \mathbf{y})=p_{t}^{(d)}(\mathbf{y}-\mathbf{x})(1+o(1))
$$

if $x \wedge y \rightarrow \infty$ under $x y \leq t$. The case when $x \wedge y<M$ is now easily dealt with by looking at (17) with $r$ suitably chosen so that $r \rightarrow \infty$ subject to $(x \vee y) r<M t$, and arguing as in the proofs of Lemma 2.2 and Theorem 3. In view of Proposition 2.2 this shows Theorem 4 ,

REMARK 2. In (36) the error estimate of the order $o\left(t^{-\eta}\right)$ with some $\eta>0$ in place of $o(1)$ can be derived in a way analogous to the proof of Theorem 3 . 


\subsection{Case $y \asymp t$ and $x=O(1), d \geq 2$}

Here we consider the case when $x \vee y \asymp t$ and $x \wedge y=O(1)$, which eludes the results stated so far.

Proposition 2.3. Let $d \geq 2$. For each $\varepsilon>0$ and $M>1$, uniformly for $\mathbf{x} \in \Omega_{A} \backslash \operatorname{nbd}_{\varepsilon}\left(A^{r}\right)$ and $\mathbf{v} \in \mathbf{R}^{d} \backslash\{0\}$ satisfying $x<M$ and $\varepsilon<|\mathbf{v}|<M$, as $t \rightarrow \infty$ and $\mathbf{y} / t \rightarrow \mathbf{v}$

$$
p_{t}^{A}(\mathbf{x}, \mathbf{y}) \sim c^{A}(\mathbf{x} ; \mathbf{v}) p_{t}^{(d)}(\mathbf{y}-\mathbf{x})
$$

where $c^{A}(\mathbf{x} ; \mathbf{v})$ is jointly continuous in $\mathbf{x}, \mathbf{v}$, positive and less than unity.

The next lemma provides a lower bound, which we need for the proof of Proposition 2.3. It is also used for the proof of Lemma 3.7 of the next section.

Lemma 2.4. For any $0<\delta<1$ and $M>1$, there exists a constant $c_{\delta, M}$ such that if $\delta t<R_{A} y<M t, x<M R_{A}$ and $\mathbf{x} \notin \operatorname{nbd}_{R_{A} \delta}\left(A^{r}\right)$, then $p_{t}^{A}(\mathbf{x}, \mathbf{y}) \geq c_{\delta, M} p_{t}^{(d)}(\mathbf{y}-\mathbf{x})$ for $t$ large enough.

Proof. Suppose $R_{A}=1$. By the conditions $\mathbf{x} \notin \operatorname{nbd}_{\delta}\left(A^{r}\right)$ and $x<M$ we have $p_{s}^{A}(\xi, \mathbf{x})>$ $c^{\prime}=c_{\delta, M}^{\prime}$ for $1 / 3<s<2 / 3, \xi \in U(1+\delta)$. Hence, putting $q_{a}^{(d)}(y, t)=P_{\mathbf{y}}\left[\sigma_{U(a)} \in d t\right] / d t$, we have for $t>1$ and $y>1+\delta$

$$
\begin{aligned}
p_{t}^{A}(\mathbf{x}, \mathbf{y}) & \geq \int_{0}^{t} q_{1+\delta}^{(d)}(y, t-s) \inf _{\xi \in \partial U(1+\delta)} p_{s}^{A}(\mathbf{x}-\xi) d s \\
& \geq c_{\delta, M}^{\prime} \int_{1 / 3}^{2 / 3} q_{1+\delta}^{(d)}(y, t-s) d s .
\end{aligned}
$$

But according to Theorem A.1 we have $q_{1+\delta}^{(d)}(y, t-s) \geq c_{\delta, M}^{\prime \prime} p_{t}^{(d)}(y) \geq c_{\delta, M}^{\prime \prime \prime} p_{t}^{(d)}(\mathbf{y}-\mathbf{x})$ for $s<2 / 3, x<M$ and $\delta t<y<M t$ and for $t$ large enough. Thus the lemma is verified.

Proof of Proposition 2.3. In [12] it is shown that there exists a measure $\lambda_{\mathbf{v}}^{A}(d \xi)$ on $\partial A$ such that $\lambda_{\mathbf{v}}^{A}(d \xi)$ depends on $\mathbf{v}$ continuously, the total measure $\lambda_{\mathbf{v}}^{A}(\partial A)$ is positive, and

$$
\left|\frac{H_{A}(\mathbf{y}, t ; \cdot)}{p_{t}^{(d)}(y) \Lambda_{\nu}(y / t)}-\lambda_{\mathbf{v}}^{A}(\cdot)\right|_{\mathrm{t} . \mathrm{var}} \longrightarrow 0
$$

(as $\mathbf{y} / t \rightarrow \mathbf{v}, t \rightarrow \infty$ ), where $\Lambda_{\nu}$ is given by (67) and $|\cdots|_{\text {t.var }}$ designates the total variation of a signed measure. Using this formula we are to compute the integral on the right side of (26) with $\mathbf{x}$ and $\mathbf{y}$ interchanged to show that for some $c^{*}=c^{*}(\mathbf{x} ; \mathbf{v})>0$,

$$
p_{t}^{(d)}(\mathbf{y}-\mathbf{x})-p_{t}^{A}(\mathbf{x}, \mathbf{y}) \sim c^{*} p_{t}^{(d)}(\mathbf{y}-\mathbf{x}) .
$$

To this end we first see that this integral restricted to $(0, t / 2]$ is

$$
\int_{0}^{t / 2} d s \int_{\partial A} H_{A}(\mathbf{y}, s: d \xi) p_{t-s}^{(d)}(\mathbf{x}-\xi) \leq \frac{C}{t^{d / 2}} P_{\mathbf{y}}\left[\sigma_{A}<t / 2\right],
$$

hence negligible, for $P_{\mathbf{y}}\left[\sigma_{A}<t / 2\right] \leq p_{t}^{(d)}(y) \times o(1)$, provided $y \asymp t$. For the rest of the integral we compute $\int_{t / 2}^{t} p_{s}^{(d)}(\mathbf{y}) p_{t-s}^{(d)}(\mathbf{x}-\xi) d s$ because of (37). Recall identity (31) and consider the integral $J$ in the proof of Lemma 2.3 with $\theta=1 / 2$ and with $\mathbf{x}$ and $\mathbf{z}$ replaced 
by $\mathbf{y}$ and $\mathbf{x}-\xi(\xi \in \partial A)$, respectively. On putting $\tilde{\mathbf{x}}=\mathbf{x}-\xi$, observe that the integral tends to concentrate on $\left[t-t^{\alpha}, t\right]$ for any $\alpha \in(0,1)$ and is asymptotic to $\int_{0}^{\infty} p_{u}^{(d)}\left(t^{-1}(\mathbf{y}-\tilde{\mathbf{x}}) u+\tilde{\mathbf{x}}\right) d u$ (provided that $y \asymp t, x<M$ and $\tilde{\mathbf{x}} \neq 0$ ). As in (32) we derive an explicit expression of this integral, which immediately leads to

$$
\int_{t / 2}^{t} p_{T}^{(d)}\left(\frac{t-s}{t} \mathbf{y}+\frac{s}{t} \tilde{\mathbf{x}}\right) d s \sim \frac{2}{(2 \pi)^{d / 2}}\left(\frac{|\mathbf{y}-\tilde{\mathbf{x}}|}{t|\tilde{\mathbf{x}}|}\right)^{\nu} K_{\nu}\left(\frac{|\mathbf{y}-\tilde{\mathbf{x}}||\tilde{\mathbf{x}}|}{t}\right) e^{-(\mathbf{y}-\tilde{\mathbf{x}}) \cdot(\mathbf{x}-\xi) / t} .
$$

Now apply formula (37). Noting $p_{t}^{(d)}(\tilde{\mathbf{x}}-\mathbf{y})=p_{t}^{(d)}(\mathbf{y}-\mathbf{x}) e^{-\mathbf{y} \cdot \xi / t}(1+O(1 / t))$, we then readily find that formula (38) holds with

$$
c^{*}=\frac{e^{-\mathbf{v} \cdot \mathbf{x}}}{K_{\nu}(|\mathbf{v}|)} \int_{\partial A} \frac{K_{\nu}(|\mathbf{v}||\mathbf{x}-\xi|)}{|\mathbf{x}-\xi|^{\nu}} \lambda_{\mathbf{v}}^{A}(d \xi) .
$$

Thus, in view of Lemma 2.4, the asymptotic formula of the proposition holds true with $c^{A}(\mathbf{x} ; \mathbf{v}):=1-c^{*} \in(0,1)$.

\section{$3 \quad$ Upper and lower bounds in the regime $x \vee y>t$}

Let $\mathbf{e}=(1,0, \ldots, 0) \in \mathbf{R}^{d}$ and suppose

$$
\mathbf{y}=-y \mathbf{e} \text { and } \quad x \leq y
$$

throughout this section. Let $\mathbf{x}=\left(x_{1}, \mathbf{x}^{\prime}\right)$, where $x_{1}=\mathbf{x} \cdot \mathbf{e} \in \mathbf{R}$ and $\mathbf{x}^{\prime}=\operatorname{pr}_{\mathbf{e}} \mathbf{x}=$ $\left(x_{2}, \ldots, x_{d}\right) \in \mathbf{R}^{d-1}$. Given $a>0$, put

$$
W=W_{a, \mathbf{e}}=\left\{\mathbf{z} \in \mathbf{R}^{d} \backslash U(a): \mathbf{z} \cdot \mathbf{e}>0,\left|\operatorname{pr}_{\mathbf{e}} \mathbf{z}\right|<a\right\}
$$

and, when $x_{1}>0$,

$$
\rho=\frac{x_{1}}{y+x_{1}} .
$$

Note that $\rho \leq 1 / 2$. We shall be concerned almost exclusively with the case $\mathbf{x} \in W$. We shall decompose $p_{t}^{A}(\mathbf{x}, \mathbf{y})$ by means of the first hitting of $L_{h}:=\left\{\left(h, \mathbf{z}^{\prime}\right): \mathbf{z}^{\prime} \in \mathbf{R}^{d-1}\right\}(h \in[0, a])$, the plane perpendicular to the vector e and passing through the point $h \mathbf{e}$.

We are primarily interested in the case when $x_{1} / y \ll 1$ and $\rho$ can be replaced by $x_{1} / y$ in the main results given below. Our choice above however is natural in view of the next lemma; its proof may be modified to show that the first hitting time of $L_{h}\left(0 \leq h<x_{1}\right)$ by the Brownian motion $\left(B_{t}\right)$ started at $\mathbf{x}$ and conditioned to be in $\mathbf{y}(=-y \mathbf{e})$ at time $t$ concentrates in a relatively small interval about $\rho t$ with a high probability if $\rho$ and $t /\left(x_{1}-h\right) y$ are small enough. The next lemma provides a crude lower bound but covers a wide range of $x_{1}$ and $y$.

Lemma 3.1. Let $X_{t}$ be a (standard) linear Brownian motion and $T_{b}$ its first passage time of $b \in \mathbf{R}$. Let $0<b<\ell$ and put $\rho_{1}=b / \ell$. Suppose $\rho_{1} \leq 1 / 2$. Then for a universal constant $c>0$,

$$
P\left[\frac{1}{2} \rho_{1} t<T_{b}<\rho_{1} t \mid X_{0}=0, X_{t}=\ell\right]>c(1 \wedge \sqrt{b \ell / t}), \quad t>0 .
$$

Proof. Let $k=\ell / t$ and $T=(t-s) s / t$. As in the proof of Lemma 2.3 the conditional probability in (39) is written as

$$
\int_{\rho_{1} t / 2}^{\rho_{1} t} p_{T}^{(1)}(k s-b) \frac{b}{s} d s
$$


Denote this integral by $J$ and observe that

$$
J=\int_{\rho_{1} t / 2}^{\rho_{1} t} \exp \left\{-\frac{k b}{2(1-s / t)}\left(\frac{k}{b} s+\frac{b}{k s}-2\right)\right\} \frac{b s^{-3 / 2} d s}{\sqrt{2 \pi(1-s / t)}} .
$$

Changing the variable of integration by $u=(k / b) s=s / \rho_{1} t$ we have

$$
J=\frac{\sqrt{k b}}{\sqrt{2 \pi}} \int_{1 / 2}^{1} \exp \left\{-\frac{k b}{2\left(1-\rho_{1} u\right)}\left(u+\frac{1}{u}-2\right)\right\} \frac{u^{-3 / 2} d u}{\sqrt{1-\rho_{1} u}} .
$$

Finally, noting $u+u^{-1}-2=(1-u)^{2} / u \leq 2(1-u)^{2}$ for $u>1 / 2$, we infer that if $\rho_{1}<1 / 2$, then $J \geq 2 \sqrt{k b} \int_{0}^{1 / 2} e^{-k b s^{2} /\left(1-\rho_{1}\right)} d s \geq 2 \int_{0}^{\frac{1}{2} \sqrt{k b}} e^{-2 s^{2}} d s \geq c(1 \wedge \sqrt{k b})$ as desired.

Denote by $q_{0}^{(1)}(x, t)$ the density of the distribution $P\left[T_{x}<t \mid X_{0}=0\right]$, given explicitly by

$$
q_{0}^{(1)}(x, t)=\frac{x}{t} p_{t}^{(1)}(x)
$$

Formula (39) will be applied in the form

$$
\int_{\rho_{1} t / 2}^{\rho_{1} t} p_{t-s}^{(1)}(\ell-b) q_{0}^{(1)}(b, s) d s \geq c\left(1 \wedge \sqrt{\frac{b \ell}{t}}\right) p_{t}^{(1)}(\ell) .
$$

Analogously to (40) we have

$$
P\left[\frac{1}{2} \rho_{1} t<T_{b}<\rho_{1} t \mid X_{0}=0, T_{\ell}=t\right]=\frac{t}{\ell} \int_{\rho_{1} t / 2}^{\rho_{1} t} p_{T}^{(1)}(k s-b) \frac{b(\ell-b)}{s(t-s)} d s .
$$

Plainly $t(\ell-b) / \ell(t-s) \geq 1-\rho_{1}$ and from the proof above we obtain

Lemma 3.2. Under the same setting and assumption as in Lemma 3.1

$$
\int_{\rho_{1} t / 2}^{\rho_{1} t} q_{0}^{(1)}(\ell-b, t-s) q_{0}^{(1)}(b, s) d s \geq c\left(1 \wedge \sqrt{\frac{b \ell}{t}}\right) q_{0}^{(1)}(\ell, t) .
$$

By the equality $p_{t-s}^{(d)}(\ell-b) p_{s}^{(d)}(b)=p_{t}^{(d)}(\ell) p_{T}^{(d)}(k s-b)$, the computation similar to that carried out for (40) leads to the following

Lemma 3.3. Let $\alpha$ be any real constant, $0<b<\ell$ and suppose $\rho_{1}:=b / \ell \leq 1 / 2$. Then for a positive constant $c>0$ that depends only on $d$ and $\alpha$,

$$
\int_{\frac{1}{2} \rho_{1} t}^{\rho_{1} t} p_{t-s}^{(d)}(\ell-b) p_{s}^{(d)}(b) s^{\alpha} d s \geq c\left(\rho_{1} t\right)^{\alpha-\nu}\left(\sqrt{\frac{t}{b \ell}} \wedge 1\right) p_{s}^{(d)}(\ell) .
$$

These lemmas, though stated here to explain the role of $\rho$ in the sequel, are used not for the upper bound given in the next subsection but only for lower bounds.

Theorem 2 follows immediately by combining Propositions 3.1 through 3.3 given below; $k(\mathbf{x})$ and $k_{*}(\mathbf{x})$ are given by (44) and (64), respectively.

In the sequel $\kappa_{\delta}, \kappa_{\delta}^{\prime}, \kappa_{\delta}^{\prime \prime}$ etc. denote positive constants that depend only on $\delta$ and $d$, while $C, C_{1}, c, c_{1}, c^{\prime}$ etc. continue to denote universal positive constants; they may vary in each occurrence of them 


\subsection{An upper bound valid for $t>0, d=2$}

Put

$$
k(\mathbf{x})=\frac{\left(\sqrt{x_{1}^{2}+\left(a-\left|\mathbf{x}^{\prime}\right|\right)^{2}}-x_{1}\right) x_{1}}{\left(a-\left|\mathbf{x}^{\prime}\right|\right)^{2}} \quad\left(\left|\mathbf{x}^{\prime}\right|<a, x_{1}>0\right) .
$$

It holds that $\sqrt{2}-1<k(\mathbf{x})<1 / 2$, for if $0<\alpha<1$, then $\sqrt{2}-1<(\sqrt{1+\alpha}-1) / \alpha<1 / 2$.

Proposition 3.1. Let $d=2$ and $k(\mathbf{x})$ be as above. Then there exists a universal constant $C$ such that for all $\mathbf{x} \in W$ and $t>0$,

$$
\frac{p_{t}^{U(a)}(\mathbf{x}, \mathbf{y})}{p_{t}^{(2)}(\mathbf{x}-\mathbf{y})} \leq C \frac{\sqrt{\rho t}}{a-\left|\mathbf{x}^{\prime}\right|} \exp \left\{-k(\mathbf{x}) \frac{\left(a-\left|\mathbf{x}^{\prime}\right|\right)^{2}}{\rho t}\right\} \text {. }
$$

Proof. We may and do suppose $a-\left|\mathbf{x}^{\prime}\right| \geq \sqrt{\rho t}$, for if not, the upper bound to be verified is trivial in view of the bound $k(\mathbf{x})<1$.

Let $L=\left\{\left(0, \xi^{\prime}\right): \xi^{\prime} \in \mathbf{R}\right\}$, the vertical axis of the plane. Then,

$$
\begin{aligned}
p_{t}^{U(a)}(\mathbf{x}, \mathbf{y}) & \leq \int_{0}^{t} \int_{L \backslash U(a)} p_{t-s}^{(2)}(\mathbf{y}-\xi) P_{\mathbf{x}}\left[B_{\sigma(L)} \in d \xi, \sigma_{L} \in d s\right] \\
& \leq \int_{0}^{t} p_{t-s}^{(2)}(\tilde{y}) P_{\mathbf{x}}\left[B_{\sigma(L)} \in L \backslash U(a), \sigma_{L} \in d s\right],
\end{aligned}
$$

where $\tilde{y}=\sqrt{y^{2}+a^{2}}$ and we have used $|\mathbf{y}-\xi| \geq \tilde{y}(\xi \in L \backslash U(a))$ for the second inequality. Note that

$$
P_{\mathbf{x}}\left[B_{\sigma(L)} \in L \backslash U(a), \sigma_{L} \in d s\right] / d s=\frac{x_{1}}{s} p_{s}^{(1)}\left(x_{1}\right) P_{\mathbf{x}}\left[\left|B_{s}^{\prime}\right|>a\right],
$$

where $B_{s}^{\prime}$ denotes the second component of $B_{s}$, and

$$
P_{\mathbf{x}}\left[\left|B_{s}^{\prime}\right|>a\right] \leq P_{0}\left[\left|B_{s}^{\prime}\right|>a-\left|\mathbf{x}^{\prime}\right|\right] \leq \frac{2 s}{a-\left|\mathbf{x}^{\prime}\right|} \cdot \frac{e^{-\left(a-\left|\mathbf{x}^{\prime}\right|\right)^{2} / 2 s}}{\sqrt{2 \pi s}} .
$$

Then putting $b=\sqrt{x_{1}^{2}+\left(a-\left|\mathbf{x}^{\prime}\right|\right)^{2}}$, we deduce that

$$
p_{t}^{U(a)}(\mathbf{x}, \mathbf{y}) \leq \frac{2 x_{1}}{a-\left|\mathbf{x}^{\prime}\right|} \int_{0}^{t} p_{t-s}^{(2)}(\tilde{y}) p_{s}^{(2)}(b) d s .
$$

Since $x_{1} \geq a-\left|\mathbf{x}^{\prime}\right|$, we have $x_{1}\left(y+x_{1}\right) / t=x_{1}^{2} / \rho t \geq 1$, or what we are interested in,

$$
\frac{b(\tilde{y}+b)}{t} \geq 1
$$

by which together with $b \leq \tilde{y}$ we apply the second half of Lemma 2.3 (with $\delta=1$ ) to see that

$$
\int_{0}^{t} p_{t-s}^{(2)}(\tilde{y}) p_{s}^{(2)}(b) d s \leq C_{1} p_{t}^{(2)}(\tilde{y}+b) \sqrt{\frac{t}{b(\tilde{y}+b)}} .
$$

Now, observing $\sqrt{t / b(\tilde{y}+b)} \leq C_{2} \sqrt{\rho t} / x_{1}$ and

$$
(\tilde{y}+b)^{2}-|\mathbf{x}-\mathbf{y}|^{2} \geq 2\left(b-x_{1}\right) y+a^{2}+b^{2}-x^{2} \geq 2\left(b-x_{1}\right)\left(y+x_{1}\right)
$$

we can conclude that for some universal constant $C$

$$
\frac{p_{t}^{U(a)}(\mathbf{x}, \mathbf{y})}{p_{t}^{(2)}(\mathbf{x}-\mathbf{y})} \leq C \frac{\sqrt{\rho t}}{a-\left|\mathbf{x}^{\prime}\right|} e^{-\left(b-x_{1}\right)\left(y+x_{1}\right) / t},
$$

hence the assertion of the proposition since $\left(b-x_{1}\right)\left(y+x_{1}\right) / t=k(\mathbf{x})\left(a-\left|\mathbf{x}^{\prime}\right|\right)^{2} / \rho t$. 


\subsection{Some lemmas in preparation for lower bounds, $d=2$}

Let $d=2$. If $Y_{t}$ is a linear Brownian motion, then for each $\lambda \in \mathbf{R}$, we have

$$
P\left[Y_{s^{\prime}}>0,0 \leq s^{\prime} \leq s \mid Y_{0}=\eta_{0}, Y_{s}=\eta\right]=1-e^{-2 \eta_{0} \eta / s} \quad\left(\eta_{0}>0, \eta>0, s>0\right)
$$

as is readily derived from the expression of transition density for $Y_{t}$ killed at the origin. For $y>0, z>0$ and $t>0$ put

$$
Q_{y}(z, t)=q_{0}^{(1)}(y, t) p_{t}^{(1)}(z)
$$

$\left(q_{0}^{(1)}(y, t)\right.$ is given in (41)). From (50) it follows that for $x=\left(x_{1}, x_{2}\right), x_{1}>0$,

$$
\frac{P_{\mathbf{x}}\left[B_{\sigma(L)}^{\prime} \in d z, \sigma_{L} \in d t ; \forall s \in[0, t], B_{s}^{\prime}>0\right]}{d z d t}=\left(1-e^{-2 x_{2} z / t}\right) Q_{x_{1}}\left(z-x_{2}, t\right) .
$$

Lemma 3.4. For any $0<\delta \leq 1$ there exists a constant $\kappa_{\delta}$ such that for $0 \leq \alpha \leq a, y>2 a$, $s>\delta a^{2}$ with ay/s $>1$, and $z>a+\delta \sqrt{a s / y}$,

$$
\frac{P_{-y \mathbf{e}}\left[B_{\sigma\left(L_{\alpha}\right)}^{\prime} \in d z, \sigma_{L_{\alpha}} \in d s, \sigma_{U(a)}>s\right]}{d z d s} \geq \kappa_{\delta} Q_{y+\alpha}(z, s) .
$$

Proof. The left side of (153) is written as

$$
\int_{-\infty}^{\infty} d \eta \int_{0}^{s} Q_{y-a}\left(\eta, s-s^{\prime}\right) \frac{P_{(-a, \eta)}\left[B_{\sigma\left(L_{\alpha}\right)}^{\prime} \in d z, \sigma_{L_{\alpha}} \in d s^{\prime}, s^{\prime}<\sigma_{U(a)}\right]}{d z}
$$

On restricting the outer integral to the half line $a(1+\delta \sqrt{s / a y}) \leq \eta<\infty$ and applying (52) this repeated integral is larger than

$$
\int_{a+\delta \sqrt{a s / y}}^{\infty} d \eta \int_{0}^{s} Q_{y-a}\left(\eta, s-s^{\prime}\right)\left(1-e^{-2 \delta^{2} a s / y s^{\prime}}\right) Q_{a+\alpha}\left(\eta-z, s^{\prime}\right) d s^{\prime}
$$

if $z>a+\delta \sqrt{a s / y}$. Putting $\rho_{1}=(a+\alpha) /(y+\alpha)$ we further restrict the inner integral to the interval $0<s^{\prime}<\rho_{1} s$ for which $a s / y s^{\prime} \geq 1 / 2$; noting $\rho_{1} \leq 2 a /(y+a)<2 / 3$ so that $s^{\prime}<\frac{2}{3} s$ (if $y>2 a$ ), without difficulty we also deduce that for $z>a+\delta \sqrt{a s / y}$,

$$
\int_{a+\delta \sqrt{a s / y}}^{\infty} p_{s-s^{\prime}}^{(1)}(\eta) p_{s^{\prime}}^{(1)}(\eta-z) d \eta \geq \kappa_{\delta}^{\prime} p_{s}^{(1)}(z)
$$

provided $s>\delta a^{2}$ and $\sqrt{a s / y}<a$. Hence, in view of (51), the repeated integral in (54) is larger than

$$
\kappa_{\delta}^{\prime} p_{s}^{(1)}(z) \int_{0}^{\rho_{1} s} q_{0}^{(1)}\left(y-a, s-s^{\prime}\right) q_{0}^{(1)}\left(a+\alpha, s^{\prime}\right) d s^{\prime} .
$$

Noting $(y-a)(a+\alpha) / s \geq(y-a) / y>1 / 2$ we apply Lemma 3.2 to see that the integral above is bounded from below by a positive multiple of $q_{0}^{(1)}(y+\alpha, s)$ as desired.

Lemma 3.5. Let $\tilde{B}_{t}=\left(X_{t}, Y_{t}\right)$ be a standard two-dimensional Brownian motion. Then, for all $k>0, \eta>0, \eta^{\prime}>-k \ell$ and $t>0$,

$$
P\left[Y_{s}>-k X_{s} \text { for } 0<s<t \mid \tilde{B}_{0}=(0, \eta), \tilde{B}_{t}=\left(\ell, \eta^{\prime}\right)\right]=1-e^{-2 \eta\left(\eta^{\prime}+k \ell\right) /\left(1+k^{2}\right) t} .
$$


Proof. From (50) we trivially obtain $P\left[B_{s}^{\prime}>0\right.$ for $\left.0<s<t \mid B_{0}=(0, y), B_{t}=\left(m, y^{\prime}\right)\right]=$ $e^{-2 y y^{\prime} / t}$ for any positive $y, y^{\prime}, m$. Let $\theta \in(0, \pi / 2)$ be such that $\tan \theta=k$. Then the identity of the lemma is the expression of this one in the coordinate system rotated by $\theta$ and shifted by $\eta \sin \theta$ along the horizontal axis, where $y=\eta^{\prime} \cos \theta, y^{\prime}=\left(\eta^{\prime}+k \ell\right) \cos \theta$ and $m^{2}+\left(y^{\prime}-y\right)^{2}=l^{2}+\left(\eta^{\prime}-\eta\right)^{2}$.

Lemma 3.6. For some universal constant $c$ and for all $\alpha>0$ and $s>0$,

$$
\int_{\alpha}^{2(\alpha \vee \sqrt{s})} p_{s}^{(1)}(x) d x \geq c\left(1 \wedge\left[\frac{s}{\alpha} p_{s}(\alpha)\right]\right) .
$$

Proof. Suppose $\alpha / \sqrt{s} \geq 3 / 2$. Then

$$
\int_{\alpha}^{2 \alpha} p_{s}^{(1)}(x) d x \geq\left[\frac{s}{x} p_{s}(x)\right]_{x=\alpha}^{2 \alpha}-\frac{s^{2}}{\alpha^{3}} p_{s}(\alpha) \geq \frac{5}{9} \frac{s}{\alpha} p_{s}(\alpha)-\frac{s}{2 \alpha} p_{s}(2 \alpha) \geq c \frac{s}{\alpha} p_{s}(\alpha) .
$$

If $\alpha / \sqrt{s}<3 / 2$, then $\int_{\alpha}^{2 \sqrt{s}} p_{s}^{(1)}(x) d x=\int_{\alpha / \sqrt{s}}^{2} p_{1}^{(1)}(x) d x \geq \frac{1}{2} p_{1}^{(1)}(2)$.

\subsection{Lower bounds for the case $\rho t>1$ and proof of Theorem 2}

Proposition 3.2. Let $d=2$. There exists a universal constant $c_{0}>0$ such that if $\mathbf{x} \in W$, $x_{1}>2 a$ and $a^{2}<t<a y$,

$$
\frac{p_{t}^{U(a)}(\mathbf{x}, \mathbf{y})}{p_{t}^{(2)}(\mathbf{x}-\mathbf{y})} \geq c_{0}\left[\frac{\sqrt{\rho t}}{a-\left|\mathbf{x}^{\prime}\right|} \wedge 1\right] \exp \left\{-\frac{\left(a-\left|\mathbf{x}^{\prime}\right|\right)^{2}}{2 \rho t}\left[1+2 a / x_{1}\right]\right\} .
$$

Proof. Let $L_{a}=\left\{\left(a, \xi_{2}\right): \xi_{2} \in \mathbf{R}\right\}$. This time we use the identity

$$
p_{t}^{U(a)}(\mathbf{x}, \mathbf{y})=\int_{0}^{t} \int_{-\infty}^{\infty} p_{t-s}^{U(a)}(\xi, \mathbf{y}) P_{\mathbf{x}}\left[B_{\sigma\left(L_{a}\right)}^{\prime} \in d \xi_{2}, \sigma_{L_{a}} \in d s\right]
$$

where $\xi=\left(a, \xi_{2}\right)$ ( $B_{t}^{\prime}$ denotes the second component of $B_{t}$ as before).

For the present purpose of obtaining a lower bound we restrict the outer integral to the interval $\frac{1}{2} \rho t \leq s \leq \frac{3}{2} \rho t$. The strong Markov property applied at the hitting time of $L$ yields

$$
p_{t-s}^{U(a)}(\xi, \mathbf{y}) \geq \int_{\frac{1}{2}(t-s) a / y}^{(t-s) a / y} d s^{\prime} \int_{a+\sqrt{(t-s) a / y}}^{\infty} Q_{y}\left(\tilde{\xi}_{2}, t-s-s^{\prime}\right) p_{s^{\prime}}^{U(a)}(\tilde{\xi}, \xi) d \tilde{\xi}_{2},
$$

where $\tilde{\xi}=\left(0, \tilde{\xi}_{2}\right)$. Put $h=\sqrt{(t-s) a / y}(\leq a)$. Since the line passing through the two points $\left(0, a+\frac{1}{2} h\right)$ and $\left(a, a-\frac{1}{2} h\right)$ does not intersect $U(a)$, by Lemma 3.5 applied with $k=h / a(<1)$ we obtain

$$
p_{s^{\prime}}^{U(a)}(\tilde{\xi}, \xi) \geq\left(1-e^{-h^{2} / 4 s^{\prime}}\right) p_{s^{\prime}}^{(2)}(\xi-\tilde{\xi}) \geq c_{1} p_{s^{\prime}}^{(1)}(a) p_{s^{\prime}}^{(1)}\left(\xi_{2}-\tilde{\xi}_{2}\right)
$$

for $s^{\prime}<(t-s) a / y$ and, by performing the integration w.r.t. $\tilde{\xi}_{2}$, that if $\xi_{2}>a$,

$$
p_{t-s}^{U(a)}(\xi, \mathbf{y}) \geq c_{1}^{\prime} p_{t-s}^{(1)}\left(\xi_{2}\right) \int_{\frac{1}{2}(t-s) a / y}^{(t-s) a / y} q_{0}^{(1)}\left(y, t-s-s^{\prime}\right) p_{s^{\prime}}^{(1)}(a) d s^{\prime}
$$


provided $s<\frac{3}{2} \rho t\left(\leq \frac{3}{4} t\right)$. Consequently, owing to Lemma 3.1 (applied with $\delta=1 / 2$ )

$$
p_{t-s}^{U(a)}(\xi, \mathbf{y}) \geq c_{1}^{\prime \prime} p_{t-s}^{(2)}(\mathbf{y}-\xi) \quad\left(\xi_{2}>a\right) .
$$

We consider separately the cases where $\left(a-\left|\mathbf{x}^{\prime}\right|\right)^{2}$ is less or not less than $\frac{3}{2} \rho t$. First suppose that $a-x_{2} \geq \sqrt{\frac{3}{2} \rho t}$ where $x_{2}=\left|\mathbf{x}^{\prime}\right|$ and restrict the inner integral in (55) to $a \leq \xi_{2} \leq 2 a$. Since $|\mathbf{y}-\xi|^{2}=(y+a)^{2}+\xi_{2}^{2}$, this yields for $a<\xi_{2}<2 a$

$$
p_{t}^{U(a)}(\mathbf{x}, \mathbf{y}) \geq c_{2} \int_{\frac{1}{2} \rho t}^{\frac{3}{2} \rho t} p_{t-s}^{(2)}(y+a) \frac{x_{1}-a}{s} p_{s}^{(1)}\left(x_{1}-a\right) P_{\mathbf{x}}\left[a \leq B_{s}^{\prime} \leq 2 a\right] d s,
$$

provided $t>a^{2}$. For $s<\frac{3}{2} \rho t$ so that $a-x_{2}>\sqrt{s}$, by Lemma 3.6

$$
P_{\mathbf{x}}\left[a \leq B_{s}^{\prime} \leq 2 a\right]=\int_{a-x_{2}}^{2 a-x_{2}} p_{s}^{(1)}\left(\xi_{2}\right) d \xi_{2} \geq \frac{c_{2}^{\prime} s}{a-x_{2}} p_{s}^{(1)}\left(a-x_{2}\right) .
$$

Let $x_{1}>2 a$ and put $\hat{y}=y+a, \hat{x}_{1}=x_{1}-a$ and $b=\sqrt{\hat{x}_{1}^{2}+\left(a-x_{2}\right)^{2}}$. Noting $\rho<b /(\hat{y}+b) \leq$ $\frac{3}{2} \rho$, we then apply Lemma 3.3 to obtain

$$
p_{t}^{U(a)}(\mathbf{x}, \mathbf{y}) \geq c_{2}^{\prime \prime} \frac{b}{a-x_{2}} \int_{\frac{1}{2} \rho t}^{\frac{3}{2} \rho t} p_{t-s}^{(2)}(\hat{y}) p_{s}^{(2)}(b) d s=c_{3} \frac{\sqrt{\rho t}}{a-x_{2}} p_{t}^{(2)}(\hat{y}+b) .
$$

Now we may proceed in the same way as in the preceding proof. Indeed, noting $|\mathbf{y}-\mathbf{x}|^{2}=$ $\hat{x}_{1}^{2}+\hat{y}^{2}+2 \hat{x}_{1} \hat{y}+x_{2}^{2}$ and $b \leq \hat{x}_{1}+\left(a-x_{2}\right)^{2} / 2 \hat{x}_{1}$, in place of (49) we have

$$
(\hat{y}+b)^{2}-|\mathbf{x}-\mathbf{y}|^{2}=2\left(b-\hat{x}_{1}\right) \hat{y}+b^{2}-\hat{x}_{1}^{2}-x_{2}^{2} \leq \frac{\left(a-x_{2}\right)^{2}}{x_{1}-a}\left(y+x_{1}\right)+O\left(\frac{1}{x_{1}^{2}}\right),
$$

which gives the lower bound of $e^{-\left[\left(a-x_{2}\right)^{2} / 2 \rho t\right] x_{1} /\left(x_{1}-a\right)}$ for $p_{t}^{(2)}(\hat{y}+b) / p_{t}^{(2)}(\mathbf{x}-\mathbf{y})$. Finally using the inequality $\left(x_{1}-a\right)^{-1}<x_{1}^{-1}\left(1+2 a x_{1}^{-1}\right)$ concludes the required lower bound.

For the case $0 \leq a-x_{2} \leq \sqrt{\frac{3}{2} \rho t}$, the restriction of the inner integral in (55) is made to the interval $a \leq \xi_{2} \leq a+4 \sqrt{\rho t}$. Applying Lemma 3.6 again we have for $\frac{1}{2} \rho t \leq s \leq \rho t$

$$
P_{\mathbf{x}}\left[a \leq B_{s}^{\prime} \leq a+4 \sqrt{\rho t}\right]=\int_{a-x_{2}}^{a-x_{2}+4 \sqrt{\rho t}} p_{s}^{(1)}(u) d u \geq c_{2}
$$

instead of (57), and a computation using (32) (with $\nu=1 / 2$ ) readily leads to the result. The details are omitted.

Lemma 3.7. Let $d \geq 2$. For any $\delta>0$, there exists a constant $\kappa_{\delta}$ such that if $x>(1+\delta) a$ and if either $\mathbf{x} \notin W$ or $\mathbf{x} \in W$ with $\rho t \geq \delta a^{2}$, then $p_{t}^{U(a)}(\mathbf{x}, \mathbf{y}) \geq \kappa_{\delta} p_{t}^{(d)}(\mathbf{y}-\mathbf{x})$.

Proof. Suppose $\mathbf{x} \in W$ and $x>(1+\delta) a$ with $\rho t>a^{2} \delta$, the case $\mathbf{x} \notin W$ being easier (see Proposition 3.3 if necessary). Writing $\mathbf{x}=\left(x_{1}, \mathbf{x}^{\prime}\right)$ (as before) we can also suppose $x_{1}>3 a$, for otherwise we have $3 t \geq \delta a y$ so that the result follows from Lemma 2.4. Let $L_{a}=\left\{\left(a, \mathbf{z}^{\prime}\right): \mathbf{z}^{\prime} \in \mathbf{R}^{d-1}\right\}$. For simplicity we suppose $\mathbf{x}^{\prime}=0$, entailing in particular $p_{t}^{(d)}(\mathbf{y}-\mathbf{x})=p_{t}^{(d)}\left(y+x_{1}\right)$. Make decomposition

$$
p_{t}^{U(a)}(\mathbf{x}, \mathbf{y})=\int_{0}^{t} \int_{d \xi \subset L_{a}} p_{t-s}^{U(a)}(\xi, \mathbf{y}) P_{\mathbf{x}}\left[B_{\sigma\left(L_{a}\right)} \in d \xi, \sigma_{L_{a}} \in d s\right],
$$


and note that

$$
P_{\mathbf{x}}\left[B_{\sigma\left(L_{a}\right)} \in d \xi, \sigma_{L_{a}} \in d s\right]=P\left[B_{s}^{\prime} \in d \xi^{\prime} \mid B_{0}^{\prime}=0\right] P\left[T_{x_{1}-a} \in d s \mid X_{0}=0\right]
$$

where $\xi=\left(a, \xi^{\prime}\right)$ and $T_{r}$ denotes the first passage time of $r$ for a linear Brownian motion $\left(X_{t}\right)$. Owing to the assumption $\rho t / a^{2}>\delta$, there exists a constant $\kappa_{\delta}^{\prime}>0$ such that

$$
P\left[2 a<\left|B_{s}^{\prime}\right|<4 \sqrt{t} \mid B_{0}^{\prime}=0\right]>\kappa_{\delta}^{\prime} \quad \text { if } \quad 2^{-1} \rho t<s<2^{-1} 3 \rho t .
$$

It also holds that if $s<\frac{3}{2} \rho t\left(<\frac{3}{4} t\right)$ and $2 a \leq\left|\xi^{\prime}\right|<4 \sqrt{t}$,

$$
p_{t-s}^{U(a)}(\xi, \mathbf{y})>c p_{t-s}^{(d)}(\mathbf{y}-\xi)=c p_{t-s}^{(1)}(y+a) p_{t-s}^{(d-1)}\left(\xi^{\prime}\right) \geq c^{\prime} p_{t-s}^{(1)}(y+a) p_{t}^{(d-1)}(0)
$$

for some $c, c^{\prime}>0$. Hence

$$
\begin{aligned}
\frac{p_{t}^{U(a)}(\mathbf{x}, \mathbf{y})}{p_{t}^{(d)}(\mathbf{y}-\mathbf{x})} & \geq \frac{\kappa_{\delta}^{\prime \prime} p_{t}^{(d-1)}(0)}{p_{t}^{(d)}\left(y+x_{1}\right)} \int_{\rho t / 2}^{\frac{3}{2} \rho t} p_{t-s}^{(1)}(y+a) P\left[T_{x_{1}-a} \in d s \mid X_{0}=0\right] \\
& =\kappa_{\delta}^{\prime \prime} P\left[\frac{1}{2} \rho t<T_{x_{1}-a}<\frac{3}{2} \rho t \mid X_{0}=0, X_{t}=y+x_{1}\right] \\
& >\kappa_{\delta}^{\prime \prime \prime}
\end{aligned}
$$

where Lemma 3.1 is used for the last inequality. This concludes the proof.

Proposition 3.3. Let $d=2$. For any $\delta>0$ there exists a constant $\kappa_{\delta}$ such that if $\mathbf{x} \in W \backslash U(a+\delta \sqrt{\rho t}), t>a^{2}$ and $a-\left|\mathbf{x}^{\prime}\right| \geq \delta \sqrt{\rho t}$, then

$$
\frac{p_{t}^{U(a)}(\mathbf{x}, \mathbf{y})}{p_{t}^{(2)}(\mathbf{x}-\mathbf{y})} \geq \frac{\kappa_{\delta} \sqrt{\rho t}}{a-\left|\mathbf{x}^{\prime}\right|} \exp \left\{-k_{*}(\mathbf{x})\left(\frac{a-\left|\mathbf{x}^{\prime}\right|}{\sqrt{\rho t}}+\delta\right)^{2}\right\}
$$

with a function $k_{*}(\mathbf{x}), \mathbf{x} \in W$ such that $\frac{1}{2}<k_{*}(\mathbf{x})<1$ and $\lim _{x_{1} \rightarrow \infty} k_{*}(\mathbf{x})=\frac{1}{2}$.

Proof. The proof is a modification of that of Proposition 3.2. Write $\mathbf{x}=\left(x_{1}, x_{2}\right)$ as before. We may suppose $x_{2} \geq 0$. Let $\mathbf{u}$ be the point of intersection where the horizontal line $\{(\eta, a): \eta \in \mathbf{R}\}$ meets the half line in the upper half plane that is issuing from $\mathbf{x}$ and tangential to the circle $\partial U(a)$. Let $u_{1}$ be the first coordinate of $\mathbf{u}$ (so that $\mathbf{u}=\left(u_{1}, a\right)$ ) and $L^{*}$ be the shift of $L:=L_{0}$ by $u_{1} \mathbf{e}: L^{*}=L_{u_{1}}=\left\{\left(u_{1}, \xi_{2}\right): \xi_{2} \in \mathbf{R}\right\}$ (see Figure 1). We decompose $p^{U(a)}(\mathbf{x}, \mathbf{y})$ by means of the first hitting of $L^{*}$ :

$$
p_{t}^{U(a)}(\mathbf{x}, \mathbf{y})=\int_{0}^{t} \int_{L^{*} \backslash U(a)} p_{s}^{U(a)}(\xi, \mathbf{x}) P_{\mathbf{y}}\left[B_{\sigma\left(L^{*}\right)} \in d \xi, \sigma_{L^{*}} \in t-d s, \sigma_{U(a)}>t-s\right] .
$$

(Here the starting site is not $\mathbf{x}$ but $\mathbf{y}$; this choice is made since our estimation of the hitting distribution of $L^{*}$ under $P_{\mathbf{x}}$ becomes crude as $\mathbf{x}$ gets close to $a \mathbf{e}$.) We restrict the range of integration to the rectangle $\left\{\left(s, \xi_{2}\right) \in[0,4 \rho t] \times[a+\delta \sqrt{\rho t}, 2 \sqrt{t}]\right\}$. We may and do suppose $\rho \leq 1 / 8$ so that $4 \rho t \leq t / 2$, otherwise $a^{2}<t<8 a^{2} / \delta^{2}$ owing to its premise and the assertion of the lemma being easily shown.

Considering the hitting of an appropriate half line emanating from the origin and arguing as in the derivation of (56) if necessary, we infer that if $s \leq 4 \rho t$ and $\xi \in L^{*}$ with $\xi_{2}>a+\delta \sqrt{\rho t}$

$$
p_{s}^{U(a)}(\xi, \mathbf{x}) \geq \kappa_{\delta}^{\prime} p_{s}^{(2)}(\mathbf{x}-\xi)
$$


for some constant $\kappa_{\delta}^{\prime}$. Hence

$$
p_{t}^{U(a)}(\mathbf{x}, \mathbf{y}) \geq \kappa_{\delta}^{\prime} \int_{0}^{4 \rho t} \int_{a+\delta \sqrt{\rho t}}^{4 \sqrt{t}} p_{s}^{(2)}(\mathbf{x}-\xi) P_{\mathbf{y}}\left[B_{t-s}^{\prime} \in d \xi_{2}, \sigma_{L^{*}} \in t-d s, \sigma_{U(a)}>t-s\right]
$$

where $\xi=\left(u_{1}, \xi_{2}\right)$. Owing to Lemma 3.4 the condition $\sigma_{U(a)}>t-s$ can be discarded from the probability in (61) if the constant factor is replaced by a smaller one. Observe that for $s<4 \rho t$ and $0<\xi_{2}<2 \sqrt{t}$,

$$
P_{\mathbf{y}}\left[B_{t-s}^{\prime} \in d \xi_{2}, \sigma_{L^{*}} \in t-d s\right] \geq c_{1} \frac{y}{t} p_{t-s}^{(2)}\left(y+u_{1}\right) d \xi_{2} d s
$$

so that the repeated integral in (61) is bounded below by a positive multiple of

$$
\frac{y}{t} \int_{0}^{4 \rho t} p_{t-s}^{(2)}\left(y+u_{1}\right) p_{s}^{(1)}\left(x_{1}-u_{1}\right) d s \int_{a+\delta \sqrt{\rho t}}^{4 \sqrt{t}} p_{s}^{(1)}\left(x_{2}-\xi_{2}\right) d \xi_{2}
$$

By $a-x_{2} \geq \delta \sqrt{\rho t}$ and $t>a^{2}$ it follows that $a+\delta \sqrt{\rho t}<2 \sqrt{t}$ and we may apply Lemma 3.6 to see that for $\left(a-x_{2}\right)^{2}<s<4 \rho t$,

$$
\int_{a+\delta \sqrt{\rho t}}^{4 \sqrt{t}} p_{s}^{(1)}\left(x_{2}-\xi_{2}\right) d \xi_{2} \geq \frac{c_{2} s}{a-x_{2}+\delta \sqrt{\rho t}} p_{s}^{(1)}\left(a-x_{2}+\delta \sqrt{\rho t}\right) .
$$

Hence, putting

$$
b^{*}:=\sqrt{\left(x_{1}-u_{1}\right)^{2}+\left(a-x_{2}+\delta \sqrt{\rho t}\right)^{2}},
$$

we have

$$
p_{t}^{U(a)}(\mathbf{x}, \mathbf{y}) \geq \frac{\kappa_{\delta}^{\prime \prime} y / t}{a-x_{2}+\delta \sqrt{\rho t}} \int_{0}^{4 \rho t} p_{t-s}^{(2)}\left(y+u_{1}\right) p_{s}^{(2)}\left(b^{*}\right) s d s .
$$

Put $\rho^{*}=b^{*} /\left(y+u_{1}+b^{*}\right)$. The condition $a-x_{2}>\delta \sqrt{\rho t}$ entails $|\mathbf{x}-\mathbf{u}|<b^{*}<2|\mathbf{x}-\mathbf{u}|$ and a simple geometric argument leads to $\frac{1}{2} x_{1}<|\mathbf{x}-\mathbf{u}|<2 x_{1}$. Obviously $u_{1}+b^{*}>x_{1} \geq a-x_{2}$ and these together verify

$$
2^{-1} x_{1} \leq b^{*} \leq 4 x_{1} \quad \text { and } \quad 2^{-1} \rho<\rho^{*}<4 \rho .
$$

Hence by Lemma 3.3

$$
\frac{\int_{0}^{4 \rho t} p_{t-s}^{(2)}\left(y+u_{1}\right) p_{s}^{(2)}\left(b^{*}\right) s d s}{p_{t}^{(2)}\left(y+u_{1}+b^{*}\right)} \geq c \rho^{*} t \sqrt{\frac{t}{\left(y+u_{1}+b^{*}\right) b^{*}}} \wedge 1 \geq c^{\prime} \frac{t}{y}\left[\sqrt{\rho t} \wedge b^{*}\right]
$$

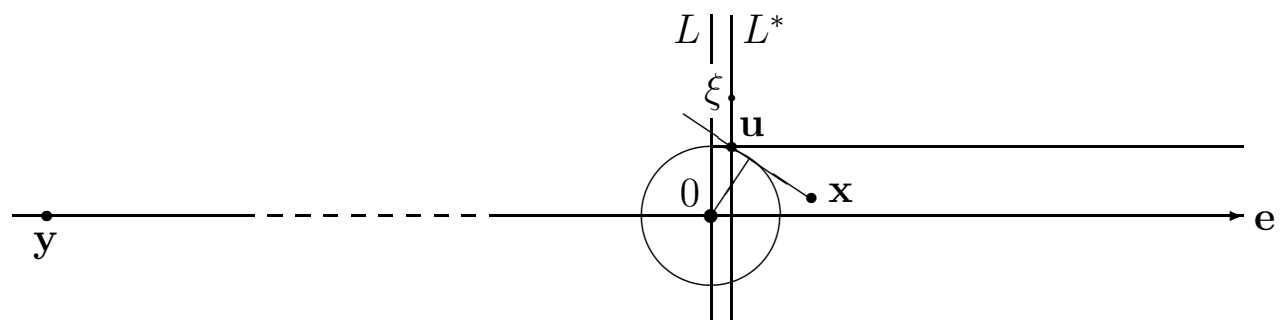

Figure 1: $\mathbf{u}$ is a point of intersection where the half line $\{(\eta, a): \eta>0\}$ meets a line passing through $\mathbf{x}$ and tangential to the circle $\partial U(a)$. 
Since $b^{*} \geq \delta \sqrt{\rho t}$, we can conclude that

$$
p_{t}^{U(a)}(\mathbf{x}, \mathbf{y}) \geq \frac{\kappa_{\delta} \sqrt{\rho t}}{a-x_{2}+\delta \sqrt{\rho t}} p_{t}^{(2)}\left(y+u_{1}+b^{*}\right) .
$$

Put

$$
k_{*}(\mathbf{x})=\frac{x_{1}}{x_{1}-u_{1}+\sqrt{\left(x_{1}-u_{1}\right)^{2}+\left(a-x_{2}\right)^{2}}} .
$$

Then

$$
\begin{aligned}
\left(y+u_{1}+b^{*}\right)^{2}-\left(y+x_{1}\right)^{2}=2 y\left(b^{*}-x_{1}+u_{1}\right)+O(1) & =2 y \frac{\left(a-x_{2}+\delta \sqrt{\rho t}\right)^{2}}{x_{1}-u_{1}+b^{*}}+O(1) \\
& \leq 2 k_{*}(\mathbf{x}) \frac{\left(a-x_{2}+\delta \sqrt{\rho t}\right)^{2}}{\rho}
\end{aligned}
$$

hence $p_{t}^{(2)}\left(y+u_{1}+b^{*}\right) / p_{t}\left(y+x_{1}\right) \geq C \exp \left\{-k_{*}(\mathbf{x})\left(a-x_{2}+\delta \sqrt{\rho t}\right)^{2} / \rho t\right\}$, which combined with (63) yields the lower bound of $p_{t}^{U(a)}(\mathbf{x}, \mathbf{y})$ asserted in the lemma. Elementary geometry shows that $\left(2 x_{1}\right)^{-1}<u_{1} \leq \sqrt{\left(x_{1}-u_{1}\right)^{2}+\left(a-x_{2}\right)^{2}}$ (with the equality only if $|\mathbf{x}|=a$ ), hence $\frac{1}{2}<k_{*}(\mathbf{x})<1$, finishing the proof.

\subsection{The higher dimensions II}

In higher dimensions $d \geq 3$ there arises a difference in the estimation of the probability

$$
P_{\mathbf{x}}\left[\left|B_{s}^{\prime}\right|>a\right]:
$$

for small $s$ this behaves differently depending on whether $a\left|\mathbf{x}^{\prime}\right|$ is larger than $s$ or not as is exhibited in Lemma 3.8 below and we accordingly need to separate the result into two cases. Let $k(\mathbf{x})$ be the function given in (44).

Theorem 5. Let $\mathbf{x} \in W \backslash U(a)$. There exist universal constants $C$ and $c>0$ such that

$$
\frac{p_{t}^{U(a)}(\mathbf{x}, \mathbf{y})}{p_{t}^{(d)}(\mathbf{x}-\mathbf{y})} \leq \begin{cases}C\left(\frac{a}{\sqrt{\rho t} \wedge a}\right)^{d-3} \exp \left\{-k(\mathbf{x}) \frac{\left(a-\left|\mathbf{x}^{\prime}\right|\right)^{2}}{\rho t}\right\}, & a\left|\mathbf{x}^{\prime}\right|<\rho t \\ C \frac{a^{\nu} \sqrt{\rho t}}{\left|\mathbf{x}^{\prime}\right|^{\nu}\left(a-\left|\mathbf{x}^{\prime}\right|\right)} \exp \left\{-k(\mathbf{x}) \frac{\left(a-\left|\mathbf{x}^{\prime}\right|\right)^{2}}{\rho t}\right\}, & a\left|\mathbf{x}^{\prime}\right| \geq \rho t\end{cases}
$$

and that if $x_{1} \geq 2 a$ and $a^{2}<t<$ ay in addition,

$$
\frac{p_{t}^{U(a)}(\mathbf{x}, \mathbf{y})}{p_{t}^{(d)}(\mathbf{x}-\mathbf{y})} \geq \begin{cases}c\left(\frac{a}{\sqrt{\rho t}}\right)^{d-3} \exp \left\{-\frac{\left(a-\left|\mathbf{x}^{\prime}\right|\right)^{2}}{2 \rho t}\left(1+\frac{2 a}{x_{1}}\right)\right\}, & a\left|\mathbf{x}^{\prime}\right|<\rho t \\ c\left[\frac{a^{\nu} \sqrt{\rho t}}{\left|\mathbf{x}^{\prime}\right|^{\nu}\left(a-\left|\mathbf{x}^{\prime}\right|\right)} \wedge 1\right] \exp \left\{-\frac{\left(a-\left|\mathbf{x}^{\prime}\right|\right)^{2}}{2 \rho t}\left(1+\frac{2 a}{x_{1}}\right)\right\}, & a\left|\mathbf{x}^{\prime}\right| \geq \rho t .\end{cases}
$$

Apparently the upper and lower bounds given above correspond to Propositions 3.1 and 3.2, respectively. Corresponding result to Proposition 3.3 (not stated) can be derived in the same way as in the proof of Theorem 5 given below (see the inequality (62) for derivation). 
Lemma 3.8. Let $\left(Y_{t}\right)$ be a Bessel process of order $\mu>-1$ and $P_{r}^{Y}$ the probability law of $Y$ started at $r \geq 0$. Then for $0 \leq r<a$,

$$
P_{r}^{Y}\left[Y_{s}>a\right]= \begin{cases}\frac{a^{2 \mu} e^{-\left(r^{2}+a^{2}\right) / 2 s}}{2^{\mu} \Gamma(\mu+1) s^{\mu}}\left(1+O\left(\frac{a^{2} r^{2}}{s^{2}} \vee \frac{s}{a^{2}}\right)\right) & \text { if } \quad \text { ar }<s, \\ \left(\frac{a}{r}\right)^{\mu+1 / 2} \frac{\sqrt{s} e^{-(a-r)^{2} / 2 s}}{a-r}\left(1+O\left(\frac{s}{(a r) \wedge(a-r)^{2}}\right)\right) & \text { if } \quad \text { ar } \geq s .\end{cases}
$$

Proof. Let $I_{\mu}$ be the modified Bessel function of the first kind of order $\mu$. Then

$$
P_{r}^{Y}\left[Y_{s}>a\right]=\int_{a}^{\infty} R_{s}(r, \eta) d \eta
$$

where for $r>0$,

$$
R_{s}(r, \eta)=\left(\frac{\eta}{r}\right)^{\mu} \frac{\eta}{s} I_{\mu}\left(\frac{r \eta}{s}\right) e^{-\left(r^{2}+\eta^{2}\right) / 2 s}
$$

and $R_{s}(0, \eta)=\lim _{r \downarrow 0} R_{s}(r, \eta)$ (cf. [[8]). Substitution from the formula

$$
I_{\mu}(y)=\left(\frac{y}{2}\right)^{\mu} \frac{1}{\Gamma(\mu+1)}\left(1+O\left(y^{2}\right)\right) \quad(0<y<1) ;=\frac{e^{y}}{\sqrt{2 \pi y}}(1+O(1 / y)) \quad(y>1)
$$

yields for $r<a<\eta$,

$$
R_{s}(r, \eta)= \begin{cases}\frac{\eta^{2 \mu+1} e^{-\left(r^{2}+\eta^{2}\right) / 2 s}}{2^{\mu} \Gamma(\mu+1) s^{\mu+1}}\left(1+O\left(\frac{(r \eta)^{2}}{s^{2}}\right)\right) & \text { if } \quad \text { ar }<s, \eta<2 a, \\ \left(\frac{\eta}{r}\right)^{\mu+1 / 2} p_{s}^{(1)}(\eta-r)\left(1+O\left(\frac{s}{r \eta}\right)\right) & \text { if } \quad \text { ar } \geq s .\end{cases}
$$

and an elementary computation of the integral in (65) leads to the formulae of the lemma (note that if $s>a^{2}$ (resp. $s>(a-r)^{2}$ ) in the case $a r<s$ (resp. ar $\geq s$ ), then the $O$ term becomes dominant so that the formula postulated in the lemma is trivial).

Proof of Theorem 5. For both the upper and lower bounds the proofs of Propositions 3.1 and 3.3 are readily adapted. Indeed by examining the proof of Lemma 2.3 we can readily obtain

$$
\int_{0}^{t} p_{t-s}^{(d)}(y) p_{s}^{(d)}(b) s^{\alpha} d s \leq C_{\alpha, d} p_{t}^{(d)}(y+b)\left(\frac{y+b}{b t}\right)^{\nu-\alpha} \sqrt{\frac{t}{b(y+b)}}
$$

For the upper bound, in the case $a\left|\mathbf{x}^{\prime}\right| \geq \rho t$ we apply Lemma 3.8 with $\mu=(d-3) / 2$ $\left(=\nu-\frac{1}{2}\right)$ and the inequality above with $\alpha=\nu, b=\sqrt{x_{1}^{2}+\left(a-\left|\mathbf{x}^{\prime}\right|\right)^{2}}$ (in place of (46) and (48), respectively), the rest being virtually unchanged. As for the case $a\left|\mathbf{x}^{\prime}\right|<\rho t$ we should take $\alpha=0, b=\sqrt{x^{2}+a^{2}}$ in (66) for which

$$
(y+b)^{2}-|\mathbf{y}-\mathbf{x}|^{2}=a^{2}+2 y\left(b-x_{1}\right) \geq \frac{2\left(a-\left|\mathbf{x}^{\prime}\right|\right)^{2}}{\rho} k(\mathbf{x})-a^{2},
$$

as is easily checked. Similar remarks apply to the lower bound. The details are omitted. 


\section{Hitting distributions}

Here we collect several results from [10, [11] and [12, the original theorems which they are taken or adapted from being indicated in the square brackets.

In the first theorem we put $\nu=\frac{1}{2} d-1$ and

$$
\Lambda_{\nu}(y):=\frac{(2 \pi)^{\nu+1}}{2 y^{\nu} K_{\nu}(y)}=\frac{2 \pi}{\int_{0}^{\infty} \exp \left(-\frac{1}{4 \pi u} y^{2}\right) e^{-\pi u} u^{\nu-1} d u}, \quad y>0,
$$

where $K_{\nu}$ is the modified Bessel function of the second kind of order $\nu$. There exists $\Lambda_{\nu}(0):=\lim _{y \downarrow 0} \Lambda_{\nu}(y) \leq \infty ; \Lambda_{0}(y) \sim-\pi / \lg y \quad$ as $y \downarrow 0$, whereas $0<\Lambda_{\nu}(0)<\infty$ for $\nu>0$.

Theorem A.1. [10, Theorem 2]. Uniformly for $x>a$, as $t \rightarrow \infty$,

$$
\begin{aligned}
\frac{P_{\mathbf{x}}\left[\sigma_{U(a)} \in d t\right]}{d t} & \sim a^{2 \nu} \Lambda_{\nu}\left(\frac{a x}{t}\right) p_{t}^{(d)}(x)\left[1-\left(\frac{a}{x}\right)^{2 \nu}\right] \\
& \sim p_{t}^{(2)}(x) \times \begin{cases}\frac{4 \pi \lg (x / a)}{(\lg t)^{2}} & (x \leq \sqrt{t}), \\
\Lambda_{0}\left(\frac{a x}{t}\right) & (x>\sqrt{t}),\end{cases}
\end{aligned}
$$

Theorem A.2. [12, Proposition 6.7]. Let $d=2$ and $\varepsilon>0$. Then, uniformly for $\mathbf{x} \in \Omega_{A} \backslash \operatorname{nbd}_{\varepsilon}\left(A^{r}\right)$, as $t \rightarrow \infty$ and $x / t \rightarrow 0$

$$
\begin{aligned}
& P_{\mathbf{x}}\left[\sigma_{A}>t\right]=\frac{2 e_{A}(\mathbf{x})}{\lg t}\left(1+O\left(\frac{1}{\lg t}\right)\right) \quad \text { for } \quad x \leq \sqrt{t} \\
& P_{\mathbf{x}}\left[\sigma_{A} \leq t\right]=\frac{1}{2 \lg (t / x)} \int_{x^{2} / 2 t}^{\infty} e^{-y} y^{-1} d y(1+o(1)) \quad \text { for } \quad x \geq \sqrt{t / \lg t}
\end{aligned}
$$

Of the results above Theorem A.1 and (i) of Theorem A.2 are applied in the proofs of Propositions 2.3 and 2.2, respectively; for all the other applications we need only the upper bounds implied by Theorem A.1 and (ii) of Theorem A.2; for the latter only the special case when $A$ is a disc (hence the assertion is essentially a corollary of Theorem A.1) is relevant. It is noted that

$$
e_{A}(\mathbf{x})=\lg (x / R)+E_{\mathbf{x}}\left[e_{A}\left(B_{\sigma_{U(R)}}\right)\right] \quad\left(x \geq R \geq R_{A}\right)
$$

(cf. [12, Lemma 6.1]) and (i) provides a better estimate than (ii) in the range $\sqrt{t / \lg t} \leq$ $x \leq \sqrt{t}$.

In the following two theorems $m_{a}$ denotes the uniform probability measure on $\partial U(a)$.

Theorem A.3. [12, Proposition 6.1]. Let $d=2$. For $r>R_{A}$ and $\mathbf{x} \in \Omega_{A} \cap U(r)$,

$$
P_{\mathbf{x}}\left[\sigma_{\partial U(r)}<\sigma_{A}\right]=\frac{e_{A}(\mathbf{x})}{\lg \left(r / R_{A}\right)}\left(1+\frac{m_{R_{A}}\left(e_{A}\right)}{\lg \left(r / R_{A}\right)}(1+\delta)\right)^{-1}
$$

with $-2\left(R_{A}^{-1} r+1\right)^{-1} \leq \delta \leq 2\left(R_{A}^{-1} r-1\right)^{-1}$. Here $m_{a}\left(e_{A}\right)=\int_{\partial U(a)} e_{A}(\xi) m_{a}(d \xi)$.

Theorem A.4. [11, Theorems 2.2 and 2.3]. Let $d \geq 2$. For $v \geq 0$, as $x / t \rightarrow v$ and $t \rightarrow \infty$,

$$
\frac{P_{\mathbf{x}}\left[B_{\sigma_{(U(1))}} \in d \xi \mid \sigma_{U(1)}=t\right]}{m_{1}(d \xi)}= \begin{cases}1+O\left(\ell_{d}(x, t) x / t\right) & \text { if } \quad v=0 \\ \psi_{v}\left(\mathcal{R}^{\mathbf{y}} \xi\right)(1+o(1)) & \text { if } \quad v>0\end{cases}
$$


uniformly for $(\xi, v) \in \partial U(1) \times[0, M]$ for each $M>1$. Here $\ell_{d}(x, t) \equiv 1$ for $d \geq 3$ and

$$
\ell_{2}(x, t)=(\lg t)^{2} / \lg (2 \vee x) \quad(x<\sqrt{t}) ; \quad=\lg (t / x) \quad(x \geq \sqrt{t}) ;
$$

$\psi_{v}(\xi)$ is a continuous function of $(v, \xi) \in[0, \infty) \times \partial U(1)$ that is positive for $v>0$ and $\psi_{0} \equiv 1 ; \mathcal{R}^{\mathbf{y}}$ denotes the rotation that sends $\mathbf{y} / y$ to the unit vector $\mathbf{e}=(1,0, \ldots, 0)$ and leaves the plane spanned by $\mathbf{e}$ and $\mathbf{y}$ invariant.

\section{References}

[1] R. F. Bass, Probabilistic techniques in analysis, Springer, 1995

[2] R. M. Blumenthal and R. K. Getoor, Markov processes and potential theory, Academic Press, 1968

[3] K. L. Chung and J. B. Walsh, Markov processes, Brownian motion, and time symmetry, 2nd ed., Springer, 2005.

[4] P. Collet, S. Martinez and J. Martin, Asymptotic behaviour of a Brownian motion on exterior domains, Probab. Theo. Rel. F. 116 (2000), 303-116.

[5] H.S. Carslaw and J.C. Jaeger, Conduction of heat in solids, Clarrendon press, Oxford, 1992.

[6] A. Erdélyi, Tables of Integral Transforms, vol. I, McGraw-Hill, Inc. (1954)

[7] N.N. Lebedev, Special functions and their applications, Prentice-Hall, Inc. (1965)

[8] D. Revuz and M. Yor, Continuous martingales and Brownian motion, 3rd ed., Springer. 1999.

[9] K. Uchiyama, Asymptotic estimates of the distribution of Brownian hitting time of a disc., J. Theor. Probab., 25 (2012), 450-463. / Erratum, J. Theor. Probab. 25 (2012), issue $3,910-911$.

[10] K. Uchiyama, Asymptotics of the densities of the first passage time distributions of Bessel diffusion, Trans. Amer. Math. Soc. 367 (2015), 2719-2742.

[11] K. Uchiyama, Density of space-time distribution of Brownian first hitting of a disc and a ball, Potential Anal. 44 (2016), 495-541.

[12] K. Uchiyama, The Brownian hitting distributions in space-time of bounded sets and the expected volume of the Wiener sausage for a Brownian bridge, available at: http://arxiv.org/abs/1406.1307v3

[13] K. Uchiyama, Asymptotic behaviour of a random walk killed on a finite set, Potential Anal (2016). doi: 10.1007/s11118-016-9598-2 